\title{
DIVERSIDAD DE LA FAMILIA POACEAE (GRAMINEAE) EN LA REGIÓN DEL BÍO-BÍO, CHILE, BASADA EN COLECCIONES DE HERBARIO
}

\section{DIVERSITY OF THE FAMILY POACEAE (GRAMINEAE) IN THE REGION OF BIO-BIO, CHILE, BASED ON HERBARIUM COLLECTIONS}

\author{
Víctor L. Finot ${ }^{1}$, Clodomiro Marticorena ${ }^{2}$, Juan A. Barrera ${ }^{1}$, \\ Mélica Muñoz-Schick ${ }^{3} \&$ María A. Negritto ${ }^{2}$ \\ ${ }^{1}$ Facultad de Agronomía, Universidad de Concepción, Casilla 537, Chillán, Chile; ${ }^{2}$ Facultad de Ciencias Naturales y \\ Oceanográficas, Universidad de Concepción, Casilla 160-C, Concepción, Chile; ${ }^{3}$ Museo Nacional de Historia Natural, \\ Casilla 787, Santiago, Chile. \\ vifinot@udec.cl
}

\section{RESUMEN}

La Región del Bío-Bío se encuentra en la zona sur de Chile central, considerado un hotspot de biodiversidad. Aunque es una región fuertemente intervenida por el hombre, principalmente por la agricultura, la forestación, la industrialización y la urbanización, tiene un bajo porcentaje de superficie protegida por el Sistema Nacional de Áreas Silvestres Protegidas del Estado (SNASPE). Por ello, documentar la biodiversidad de la región es una tarea ineludible. Se analizó la diversidad de la familia Poaceae en la Región del Bío-Bío, a partir de las bases de datos de los herbarios de la Universidad de Concepción (CONC) y del Museo Nacional de Historia Natural (SGO), así como de la literatura taxonómica. Sobre la base de esta información, se preparó una lista revisada de las especies y se confeccionaron mapas que muestran la intensidad de las recolecciones realizadas y la riqueza de especies. Para evaluar la exhaustividad del inventario se confeccionó una curva de rarefacción y se calculó la riqueza específica estimada mediante los estimadores no paramétricos Chao-2 e ICE. La flora de gramíneas de la Región del Bío-Bío, identificada a partir de 3.069 especímenes, se compone de 234 especies y 11 taxones infraespecíficos, distribuidos en 76 géneros y 8 subfamilias. Un $66,2 \%$ de las especies (155 especies) son nativas, de las cuales un 11,5\% (27 especies) son endémicas de Chile y un 54,7\% (128 especies) son nativas no endémicas; un 33,7\% (79 especies) son introducidas. Las subfamilias mejor representadas en número de especies son Pooideae (73,08\%), Panicoideae (12,39\%), Danthonioideae $(5,98 \%)$ y Chloridoideae $(4,70 \%)$. Los valores de riqueza estimada sugieren que el inventario de Poaceae se ha completado en $83-85 \%$, lo que implica que alrededor de 40 especies podrían aún ser reconocidas en la Región del Bío-Bío.

Palabras Clave: Riqueza específica, riqueza estimada, estimador de riqueza Chao-2, estimador de riqueza ICE, curva de acumulación de especies.

\section{ABSTRACT}

The Region of Bío-Bío is located on the southern edge of central Chile, considered a hotspot of biodiversity. Although the region is strongly disturbed by human activities, mainly agriculture, forestry, industrialization and urbanization, it has a low percentage of area covered by the National System of Protected Areas (SNASPE). Thus, to document the regional biodiversity is a critical task. The diversity of the family Poaceae (Gramineae) in the Region of Bío-Bío, Chile, was studied on the basis of data obtained from the databases of the herbaria of the University of Concepción (CONC) and the National Museum of Natural History (SGO), as well as from the taxonomic literature. Maps showing the collecting intensity and species richness were made. To assess the completeness of the inventory a rarefaction curve was constructed and the estimated species richness was calculated by the non-parametric indices Chao 2 and ICE. The grass flora of the Region of Bío-Bío, identified from 3,069 specimens, consists of 234 species and 11 infraspecific taxa, distributed in 76 genera and 8 subfamilies. A total of $66.2 \%$ of the species are native ( 155 species), including $11.5 \%$ (27 species) endemic from Chile and 54.7\% (128 species) native from Chile but not endemic; $33.7 \%$ (79 species) are introduced. The subfamilies better represented in number of species are Pooideae $(73.08 \%)$, Panicoideae $(12.39 \%)$, Danthonioideae (5.98\%) and Chloridoideae (4.70\%). The values of estimated richness suggest that the inventory of Poaceae has been completed in $83-85 \%$, which means that about 40 species could still be recognized in the Region of Bío-Bío.

KEYwORDS: Species richness, estimated species richness, Chao-2 richness estimator, ICE richness estimator, species accumulation curve. 


\section{INTRODUCCION}

La información sobre la biodiversidad (número de especies, clasificación taxonómica, ciclos de vida, origen geográfico, endemismos, distribución, etc.), a distintas escalas (continente, país, regiones administrativas, reservas, parques nacionales $u$ otras), constituye la base para su conservación y para el establecimiento de planes de manejo sustentables (Smith \& Wolfson 2004, Klopper et al. 2007). En la última década, los herbarios y museos han hecho un esfuerzo enorme para digitalizar las colecciones y construir bases de datos, que mantienen constantemente actualizadas. La información contenida en estas bases de datos es una herramienta imprescindible para documentar y analizar la diversidad (Funk et al. 1999, Escalante et al. 2000, Pysek 2003, Pysek et al. 2004, Morrone et al. 2005, Schmidt et al. 2005, Moreira-Muñoz \& Muñoz-Schick 2007, Bette et al. 2009).

Listas de las especies de Poaceae presentes en diferentes zonas del país han sido elaboradas por Reiche (1903), Muñoz (1941), Kunkel (1968), Marticorena \& Quezada (1985), Marticorena (1990), Arroyo et al. (1989, 1990, 1992, 1998), Matthei et al. (1993), Teillier et al. (1994), Rundel et al. (1996), Marticorena et al. (1998a,b), Baeza et al. (1999, 2002, 2007), Domínguez et al. (2006), Rodríguez et al. (2008a,b), entre otras. En Chile, la familia Poaceae comprende 544 especies (Zuloaga et al. 2008), es decir, aproximadamente un 10,1\% de la flora del país, siendo superada tan sólo por la familia Asteraceae que comprende cerca de 863 especies (Moreira-Muñoz \& Muñoz-Schick 2007). Un $60,2 \%$ de las especies son nativas no endémicas (328 especies); 68 especies (12,5\%) son endémicas y 148 especies $(27 \%)$ son introducidas (Zuloaga et al. 2008). La mayoría de las especiesintroducidas está naturalizada en Chile y presenta carácter de maleza (Matthei 1995). De las 13 subfamilias de Poaceae actualmente reconocidas (Judziewicz et al. 2000, Grass Phylogeny Working Group 2001, Peterson et al. 2001, Soreng et al. 2003, Zuloaga et al. 2003, Sánchez-Ken et al. 2007), ocho se encuentran presentes en Chile: Aristidoideae, Arundinoideae, Bambusoideae, Chloridoideae, Danthonioideae, Ehrhartoideae, Panicoideae y Pooideae. Por otra parte, en los últimos años se ha realizado un esfuerzo importante para mejorar la clasificación genérica de las especies, lo que ha derivado en la proposición de varios nuevos géneros, como por ejemplo, Amelichloa Arriaga et Barkworth (Arriaga \& Barkworth 2006), Anatherostipa (Hack. ex Kuntze) Peñailillo (Peñailillo 1996), Kikuyuochloa H. Scholz (Scholz 2006), Nicoraepoa Soreng et L.J. Gillespie (Soreng \& Gillespie 2007) y Pappostipa (Speg.) Romaschenko, P.M. Peterson et Soreng (Romaschenko et al. 2008), entre otros. Es así que, de los 109 géneros originalmente citados para Chile continental (Marticorena \& Quezada 1985, Marticorena 1990) podemos aumentar este número, en forma preliminar, a 125. Estos géneros se distribuyen en las subfamilias presentes en Chile, como sigue: Aristidoideae (1 gen.), Bambusoideae (1 gen.), Ehrhartoideae (1 gen.), Arundinoideae (2 gen.), Danthonioideae (4 gen.), Chloridoideae (18 gen.), Panicoideae (24 gen.) y Pooideae (74 gen.).

Dos consideraciones hacen particularmente interesante analizar la flora de gramíneas de la Región del Bío-Bío. En primer lugar, es una zona de transición entre la zona esclerófila mediterránea de Chile central y la zona templado-lluviosa de la Provincia Subantártica (Villagrán \& Le-Quesne 1996, Teneb et al. 2004), situada en la zona sur de Chile central, considerado un hotspot de biodiversidad (Arroyo 1999, Myers et al. 2000). En segundo lugar, es una de las zonas más pobladas del país, con un alto grado de intervención antrópica derivada de la urbanización y de la actividad industrial, agrícola y forestal; no obstante, cuenta con un bajo porcentaje de superficie protegida por el SNASPE (Vergara et al. 2006). De ahí, entonces, que resulte imperativo documentar la diversidad biológica de la Región, antes que los niveles de alteración de los ecosistemas hagan imposible su conocimiento y su conservación.

Los objetivos de este trabajo fueron elaborar una lista revisada de las especies y analizar la exhaustividad del inventario y la riqueza de especies de Poaceae en la Región del Bío-Bío, Chile, sobre la base del material hasta ahora colectado y disponible en los principales herbarios nacionales.

\section{MATERIALES Y METODOS}

Area Estudiada

La Región del Bío-Bío (36 $06^{\circ}-38^{\circ} 30^{\prime} \mathrm{S}$; $71^{\circ} 00^{\prime}$ $73^{\circ} 55^{\prime} \mathrm{W}$ ), situada entre las regiones del Maule y de la Araucanía, ocupa una superficie aproximada 
de $37.000 \mathrm{~km}^{2}$, esto es, cerca de un $5 \%$ de la superficie nacional. Se divide en 54 comunas y cuatro provincias (Arauco, Bío-Bío, Concepción y Nuble). Presenta una transición climática que se manifiesta en un aumento progresivo de la pluviometría y, en menor grado, una disminución de la temperatura en sentido norte-sur (Di Castri \& Hajek 1976). En la zona norte predomina el clima templado-mediterráneo, con 4-5 meses secos en verano y precipitaciones cercanas a los $1.000 \mathrm{~mm}$ anuales. En la Cordillera de los Andes, por sobre los $1.500 \mathrm{~m}$, el clima es frío y las precipitaciones anuales superan los $2.000 \mathrm{~mm}$. Por su parte, hacia la Cordillera de la Costa el clima es templadohúmedo y las precipitaciones fluctúan entre $1.200 \mathrm{y}$ $2.000 \mathrm{~mm}$ anuales, dependiendo de la latitud. En la zona sur de la región y especialmente hacia la costa, el clima se hace más lluvioso, con precipitaciones que alcanzan los $2.900 \mathrm{~mm}$ anuales. En el relieve regional se distinguen todas las unidades del relieve nacional, incluyendo la Cordillera de la Costa, la Depresión Intermedia y la Cordillera de los Andes. Las planicies litorales alcanzan escaso desarrollo (unos $25 \mathrm{~km}$ en la zona de Arauco). Según Gajardo (1994), en la zona costera se desarrollan el Bosque Caducifolio de Concepción y el Bosque Alto-Montano de Nahuelbuta como principales formaciones vegetales y al norte de esta zona, el Bosque Caducifolio Maulino. Los suelos de este sector (Stolpe 2006) derivan de sedimentos marinos. Las formaciones dominantes son terrazas marinas y pizarras, con algunas dunas litorales desarrolladas principalmente hacia el sur de la región (Veit \& Garleff 1995). Hacia el interior, el matorral espinoso del secano interior o espinal de Acacia caven se desarrolla principalmente sobre suelos graníticos y rojo arcillosos (Stolpe 2006). La Depresión Intermedia está principalmente ocupada por tierras agrícolas; sobre las dunas continentales se extiende hacia el sur de la región el Bosque Esclerófilo de los Arenales. La formación más importante de dunas continentales se extiende entre el río Itata por el norte y Rarinco por el sur (Stolpe 2006). El sector más oriental está ocupado por bosques caducifolios: Bosque Caducifolio de la Frontera, Bosque Caducifolio Andino de Chillán, muy fragmentado, y el Bosque Caducifolio Andino de Bío-Bío. Estas formaciones se desarrollan sobre suelos derivados de depósitos de cenizas volcánicas, denominados "trumaos" (Veit \& Garleff 1995, Stolpe 2006). En el sur-este se encuentra el Bosque Caducifolio Alto Andino de la Araucanía. Finalmente, la Estepa Alto Andina ocupa el límite cordillerano oriental (Gajardo 1994, Stolpe 2006). Cabe mencionar que el 91,5\% de los suelos de la región presentan algún grado de erosión (muy grave, grave o moderada), distribuida principalmente en la Cordillera de la Costa y planicies litorales asociadas, depresión intermedia y precordillera andina (IREN 1979).

El territorio insular de la región incluye las islas Quiriquina, Mocha y Santa María. Los sectores protegidos por el Estado son, en el sector andino, la Reserva Nacional Huemules de Niblinto, Reserva Nacional Ñuble, Reserva Nacional Ralco y Parque Nacional Laguna del Laja. El territorio insular de la región incluye las islas Quiriquina, Mocha y Santa María. Los sectores protegidos por el Estado son, en el sector andino, la Reserva Nacional Huemules de Niblinto, Reserva Nacional Nuble, Reserva Nacional Ralco y Parque Nacional Laguna del Laja. En el sector costero, se encuentra la Reserva Nacional Isla Mocha, los Santuarios de la Naturaleza Península de Hualpén y Lobería de Cobquecura, y el parque educativo privado Coyanmahuida.

\section{Métodos}

Se preparó una base de datos (BD) a partir de la información de las bases de datos de los herbarios CONC (Herbario Universidad de Concepción, preparada por C. Marticorena), SGO (Herbario del Museo Nacional de Historia Natural, Santiago, preparada por M. Muñoz-Schick), herbario de la Facultad de Agronomía de la Universidad de Concepción (CONC-CH) y especímenes citados en revisiones taxonómicas previamente publicadas [Acevedo de Vargas (1959, Cortaderia Stapf); Baeza (1996, Danthonia DC., Rytidosperma Steud.); Cialdella \& Arriaga (1998, Piptochaetium J.Presl); Cialdella \& Giussani (2002, Piptochaetium); De Paula (1975, Anthoxanthum L. bajo Hierochlöe R.Br.); Finot et al. (2005, Trisetum Pers.); Matthei (1965, Nassella (Trin.) E.Desv. bajo Stipa L.; 1975, Briza L.; 1986, Bromus L.; 1987a, Aristida L.; 1987b, Panicum L.); Muñoz-Schick (1985, Melica L.; 1990, Nassella); Nicora (1998, Eragrostis Wolf); Rúgolo de Agrasar (1978, Calamagrostis Adans. bajo Deyeuxia Kunth; 1982, Bromidium Nees et Meyen; 1999, Corynephorus P.Beauv.); Rúgolo de Agrasar \& Molina (1997, Agrostis L.); Soreng \& Peterson (2008, Poa L.)].

Se incorporaron 3.069 registros en la $\mathrm{BD}$; para 
cada registro se anotó el nombre de la especie o taxón subespecífico, subfamilia, tribu, origen, recolector, número de recolector, latitud, longitud, altitud, localidad, mes y año de recolección, herbario y número de herbario. A partir de la BD se preparó un listado de las especies presentes (Tabla I), siguiendo la clasificación propuesta por la base de datos TROPICOS de Missouri Botanical Garden, disponible en Internet (http://www. tropicos.org), Catalogue of New World Grasses (CNWG) (Judziewicz et al. 2000; Peterson et al. 2001; Soreng et al. 2003; Zuloaga et al. 2003) y GPWG (2001). Para cada especie se anotó el origen geográfico como endémica, nativa no endémica o introducida, el ciclo de vida como anual, bienal o perenne, la distribución en la región por provincias, la presencia en países limítrofes, la altitud incluyendo los límites inferior y superior y la distribución en Chile por regiones (Tabla I). La distribución de las especies en países limítrofes se consultó en las bases de datos TROPICOS (loc. cit.) e IRIS (http://www.darwin.edu.ar/ Proyectos/ FloraArgentina) disponibles en Internet y de la literatura pertinente (Hitchcock 1927, Tovar 1957, 1960, Nicora 1978, Nicora \& Rúgolo de Agrasar 1987, Renvoize 1998). En esta lista se entregan los nombres completos de todos los taxones, incluyendo sus autores. Se confeccionaron mapas de distribución de las recolecciones, de la riqueza de especies y de la distribución de las especies endémicas. Para ello, la Región del Bío-Bío se dividió en 86 cuadrículas de 0,25 x 0,25 grados de latitud y longitud, utilizando el programa Arc View 3.2 (ESRI 1996).

Para evaluar la exhaustividad del inventario representado por las colecciones de los herbarios analizadas, se construyó una curva de acumulación de especies, utilizando el programa EstimateS 8.0 (Colwell et al. 2004, Colwell 2006). Como medida del esfuerzo de recolección se consideraron las muestras realizadas en el tiempo (especies recolectadas por década en las 18 décadas comprendidas entre 1828 y 2007). La riqueza estimada se calculó utilizando dos tipos de coeficientes: un coeficiente paramétrico basado en la función de Clench (Soberón \& Llorente 1993, León et al. 1998) utilizando el programa Infostat 2009 (Di Rienzo et al. 2009) y los índices no paramétricos Chao-2 e ICE utilizando el programa EstimateS 8.0 (Moreno \& Halffter 2000, JiménezValverde \& Hortal 2003, Colwell 2006).

\section{RESULTADOS}

EXHAUSTIVIDAD DEL INVENTARIO DE LA FAMILIA Poaceae en la Región del Bío-Bío

La BD analizada incluye material recolectado en una etapa bastante temprana del conocimiento de la diversidad de plantas en Chile, que comprende las colecciones realizadas por Poeppig (1828 y 1832), Gay (1839), De Solís (1860-1864), Rodulfo Amando y Federico Philippi (1861-1894), Kunze (1877), Reiche (1890-1909), Neger (1895) y Dusén (1896), entre otras, hasta el año 2007. El número de especímenes y consecuentemente de especies recolectados en las primeras 10 décadas (1828-1917) es relativamente bajo (Fig. 1). En este período fueron recolectados entre 1 y 49 especímenes por década que representan un total de 85 especies, cerca de un 36\% del total de especies actualmente registradas en la Región. A partir de 1918, el esfuerzo de recolección se incrementó notablemente, recolectándose entre 119 y 605 especímenes por década, aumento que se traduce en 151 especies de Poaceae no registradas previamente en la Región. La distribución de las recolecciones no es homogénea en la Región (Fig. $2)$, por el contrario, algunas áreas concentran la mayor parte de ellas. Las zonas más recolectadas son los alrededores de Chillán $\left(36^{\circ} 36^{\prime} \mathrm{S}, 72^{\circ} 05^{\prime} \mathrm{W}\right)$, la cordillera de Chillán (Termas de Chillán, Valle de Aguas Calientes, Valle de las Nieblas) (36 $54^{\circ}$ 'S, $71^{\circ} 24^{\prime} \mathrm{W}$ ), el Parque Nacional Laguna del Laja $\left(37^{\circ} 21^{\prime} \mathrm{S}, 7^{\circ} 22^{\prime} \mathrm{W}\right)$ y los alrededores de Concepción ( $\left.36^{\circ} 50^{\prime} \mathrm{S}, 73^{\circ} 03^{\prime} \mathrm{W}\right)$. Las cuadrículas más intensamente recolectadas son la $n^{0} 24$ con 644 especímenes (Concepción), la n ${ }^{\circ} 31$ con 144 especímenes (Cordillera de Chillán), la $n^{\circ} 18$ con 134 especímenes (Chillán) y la $\mathrm{n}^{\circ} 53$ con 114 especímenes (P.N. Laguna del Laja). También se observa la existencia de cuadrículas no colectadas, especialmente en la zona sur de la región (cuadrículas 4, 5, 12, 13, 23, 69, 70, 71, 74, 77, $78,79,81,83,85$ y 86). La curva de acumulación de especies (Fig. 3) muestra el número de especies recolectadas en función del esfuerzo de muestreo (tiempo). Esta curva muestra que, si bien no se ha registrado la totalidad de las especies, el inventario realizado es confiable. La riqueza específica estimada a partir de la asíntota de la curva ( $\mathrm{y}=$ $71,45 \mathrm{x} / 1+0,26 \mathrm{x})$ es de 274,8 , indicando que el inventario se ha completado en un $85 \%$ y que aún sería posible detectar unas 40 especies más en la 
Gayana Bot. 66(2), 2009

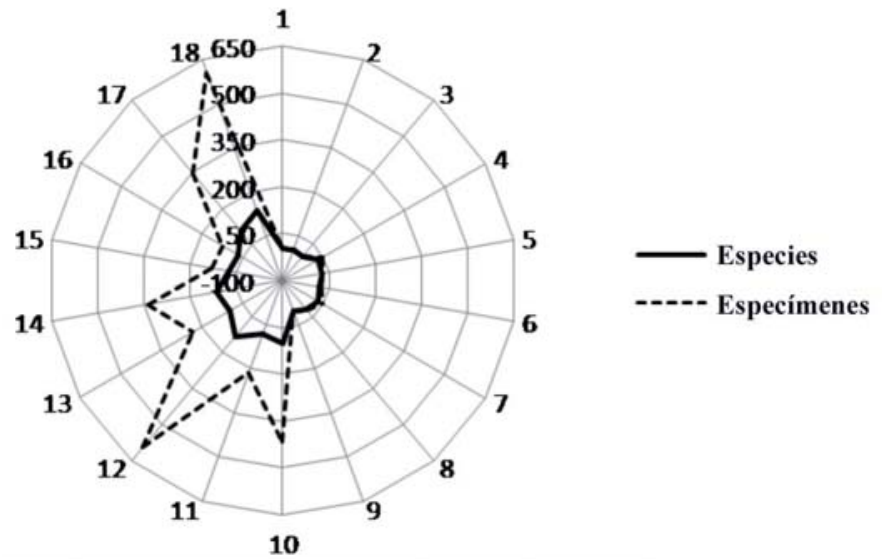

Figura 1. Número de especímenes y número de especies recolectados en la Región del Bío-Bío, en las 18 décadas comprendidas entre 1828 y 2007.

FiguRE 1. Number of specimens and number of species collected in Bío-Bío Region in the 18 decades between 1828 and 2007.

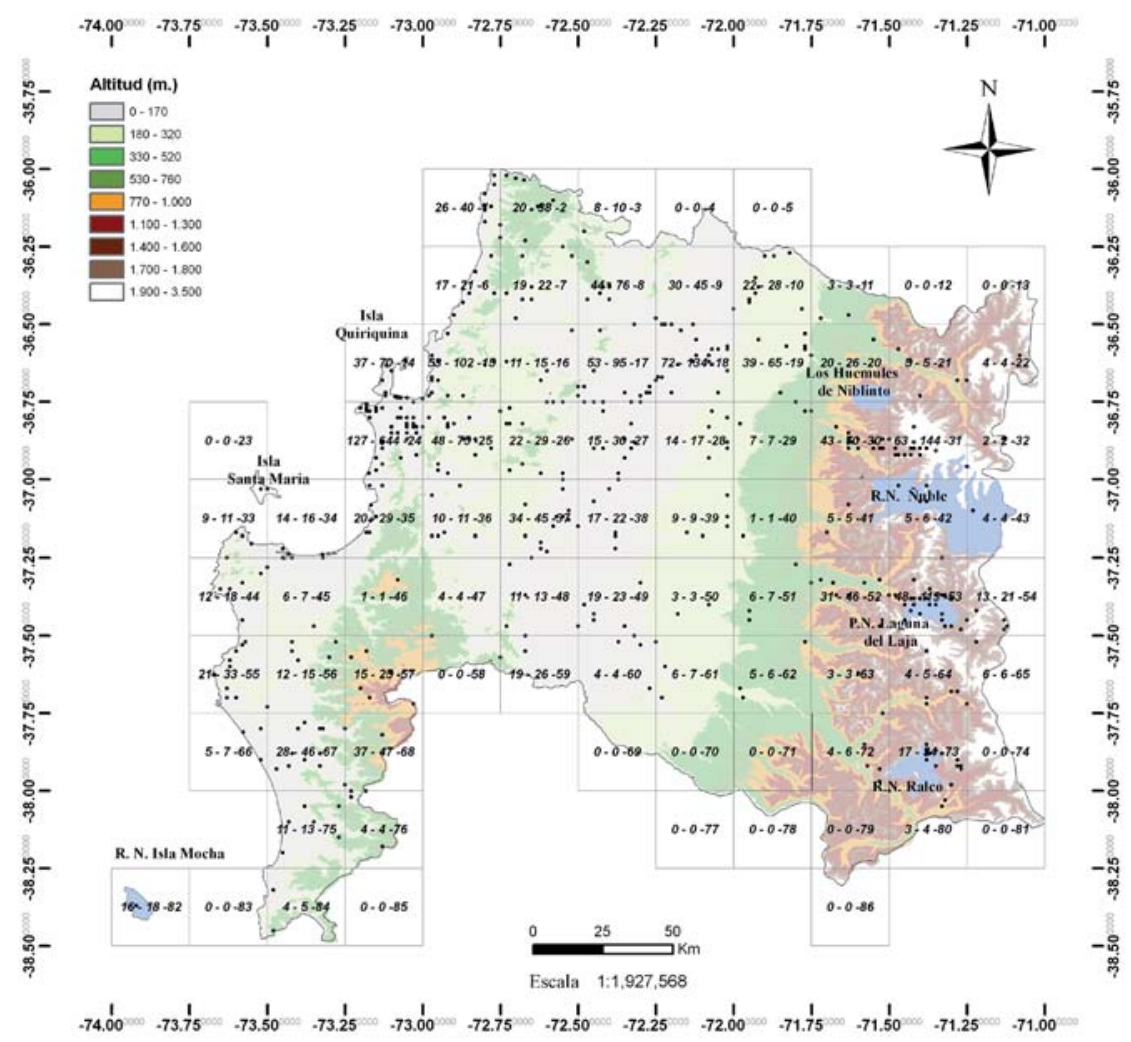

Figura 2. Mapa de las localidades recolectadas en la Región del Bío-Bío. Cada punto representa al menos un especimen.

Figure 2. Map of the localities collected in the Region of Bío-Bío. Each point represents at least one specimen. 


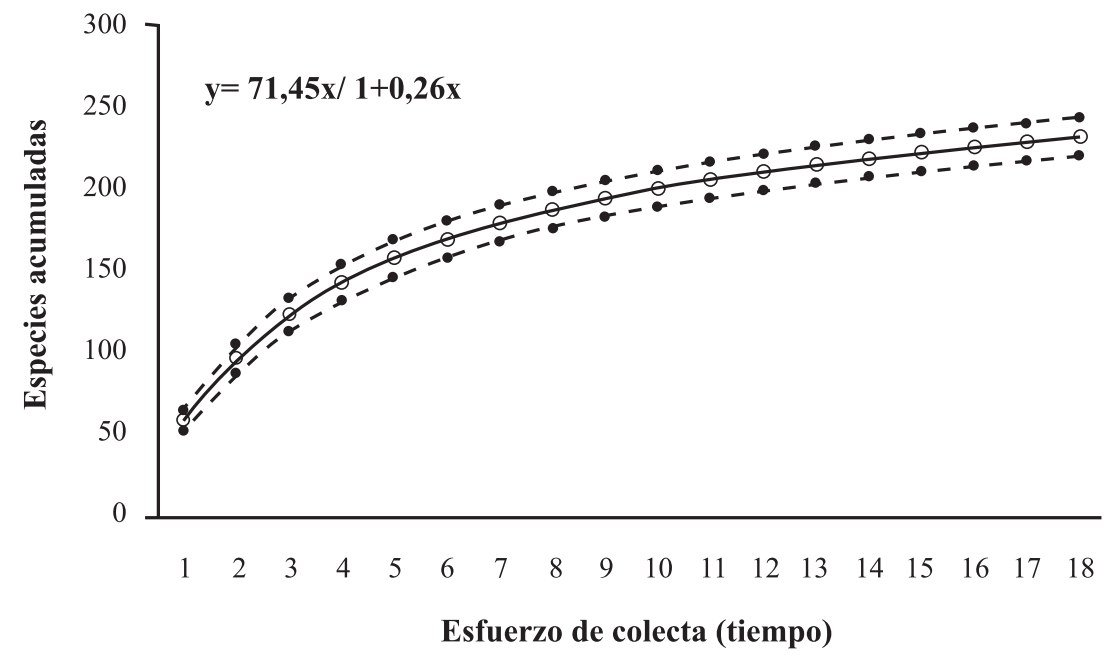

Figura 3. Curva de acumulación de especies (línea continua), basada en las especies recolectadas en la Región del BíoBío, en las 18 décadas comprendidas entre 1828 y 2007, con intervalos de confianza de $95 \%$ (líneas de puntos).

FIGURE 3. Species accumulation curve (solid line) based on collected species between 1828 and 2007 (18 decades), in the Region of Bío-Bío, with 95\% confidence intervals (dotted lines).

zona estudiada. Por otra parte, la riqueza específica estimada mediante los índices ICE y Chao-2 es de 264.89 y 268.95 , respectivamente. Estos valores sugieren que el inventario se ha completado entre 87 y $88 \%$ y por lo tanto, entre 30 y 35 especies podrían aún ser detectadas en la región.

COMPOSICIÓN TAXONÓMICA Y RIQUEZA DE ESPECIES

Doscientas treinta y cuatro especies y 11 taxa infraespecíficos, distribuidos en 76 géneros y ocho subfamilias, fueron identificados a partir de los 3.069 especímenes ingresados a la BD (Tabla I). El número de tribus, géneros y especies registrados de cada subfamilia están resumidos en la Tabla II. Las ocho subfamilias presentes en Chile están representadas en la Región: Aristidoideae (2 spp., 0,85\%), Arundinoideae (1 sp., 0,43\%), Bambusoideae (5 spp., 2,14\%), Chloridoideae (11 spp., 4,70\%), Danthonioideae (14 spp., 5,98\%), Ehrhartoideae (1 sp., 0,43\%), Panicoideae (29 spp., 12,39\%) y Pooideae (171 spp., 73,08\%). Las especies se distribuyen en 14 tribus, de las cuales seis pertenecen a Pooideae y dos a Panicoideae. Las seis subfamilias restantes comprenden sólo una tribu cada una. La subfamilia mejor representada es Pooideae, con un
$73,08 \%$ de las especies y un $67 \%$ de los géneros (171 spp. de 51 gén.). En Pooideae, los géneros que presentan el mayor número de especies son Agrostis L. (19 spp.), Nassella (Trin.) E.Desv. (15 spp.), Bromus L. (14 spp.), Poa L. (14 spp.) y Polypogon Desf. (incl. Chaetotropis Kunth, 10 spp.) (Tabla I). La subfamilia Panicoideae es la segunda en importancia, con un $12,39 \%$ de las especies y un $16 \%$ de los géneros (29 especies de 12 géneros) (Tabla II). En Panicoideae, los géneros mejor representados son Panicum L. (5 spp.), Paspalum L. (5 spp.) y Setaria P. Beauv. (4 spp.) (Tabla I). Danthonioideae, con un 6\% de las especies y cerca de $4 \%$ de los géneros (14 especies de 3 géneros), está representada por Cortaderia Stapf (5 spp.), Danthonia DC. (4 spp.) y Rytidosperma Steud. (5 spp.) (Tabla I). Chloridoideae comprende un 5\% de las especies y casi $8 \%$ de los géneros (12 especies, 6 géneros), de los cuales Eragrostis Wolf es el que presenta la mayor cantidad de especies (5 spp.) (Tabla I). Aristidoideae, Arundinoideae, Ehrhartoideae y Bambusoideae sólo comprenden un género cada una. Bambusoideae es la que comprende la mayor cantidad de especies (5 spp.), todas incluidas en el género Chusquea Kunth (Tabla I). 
Gayana Bot. 66(2), 2009

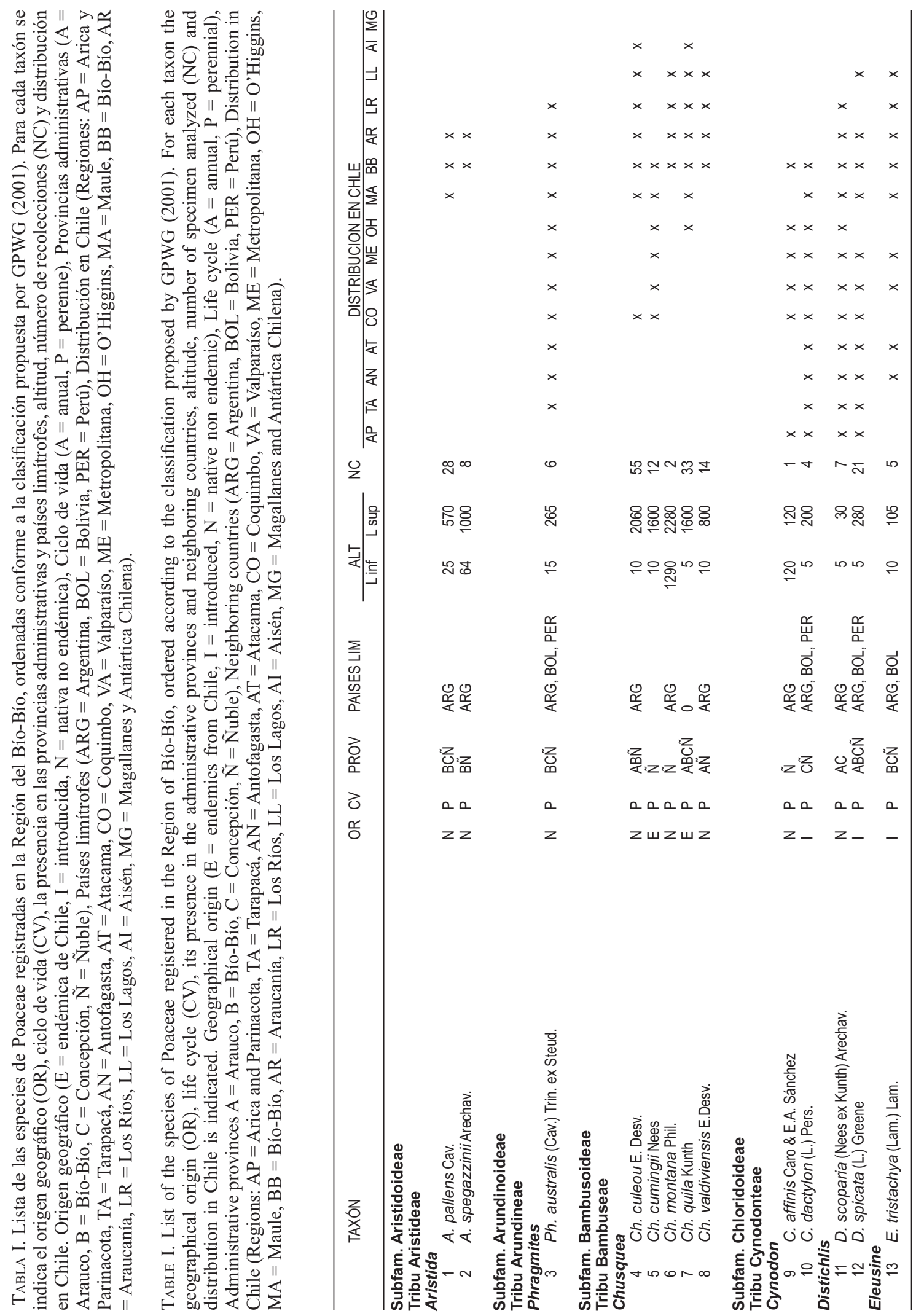


Poaceae de la Región del Bío-Bío, Chile: Finot, V.L. ET AL.

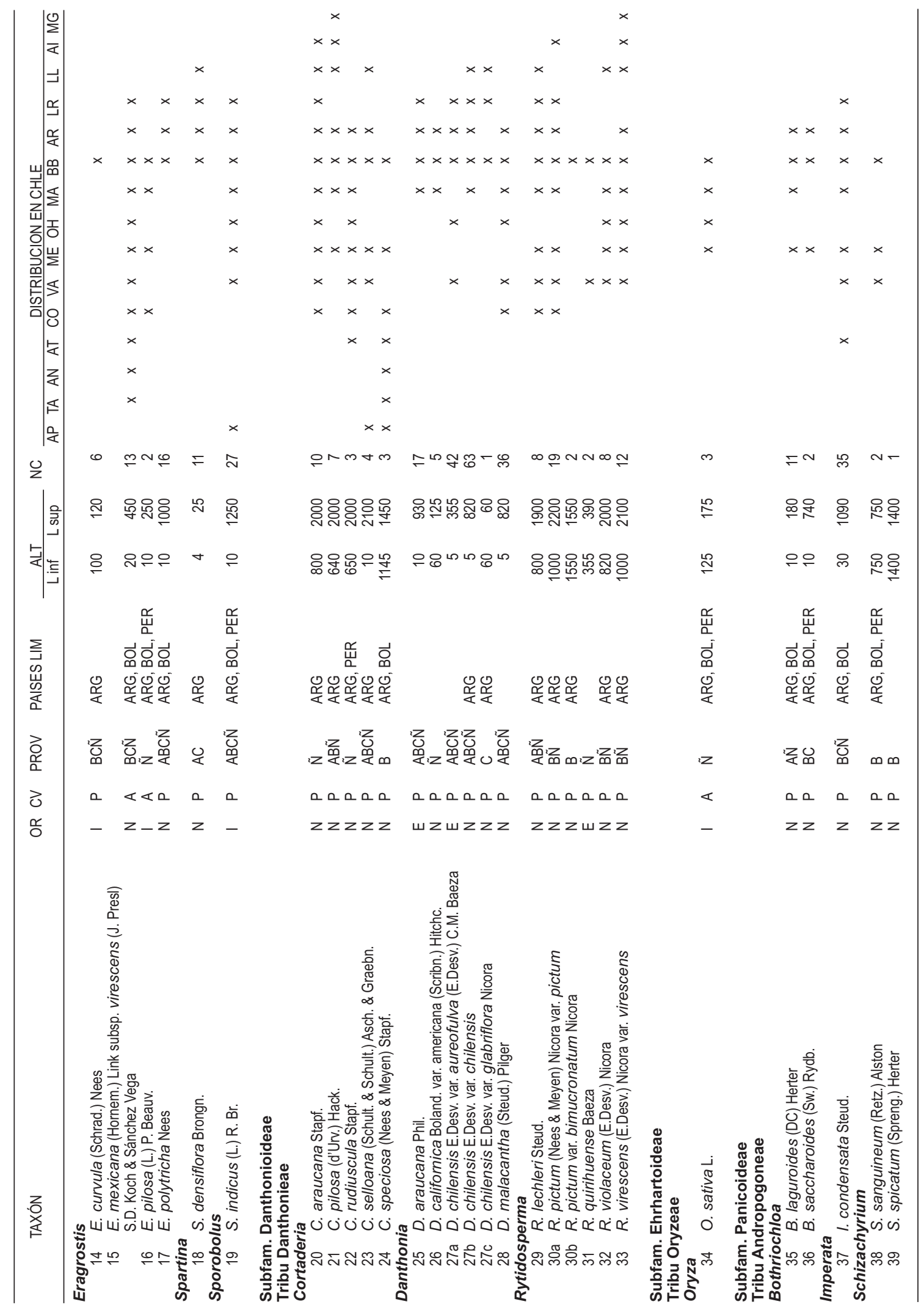


Gayana Bot. 66(2), 2009

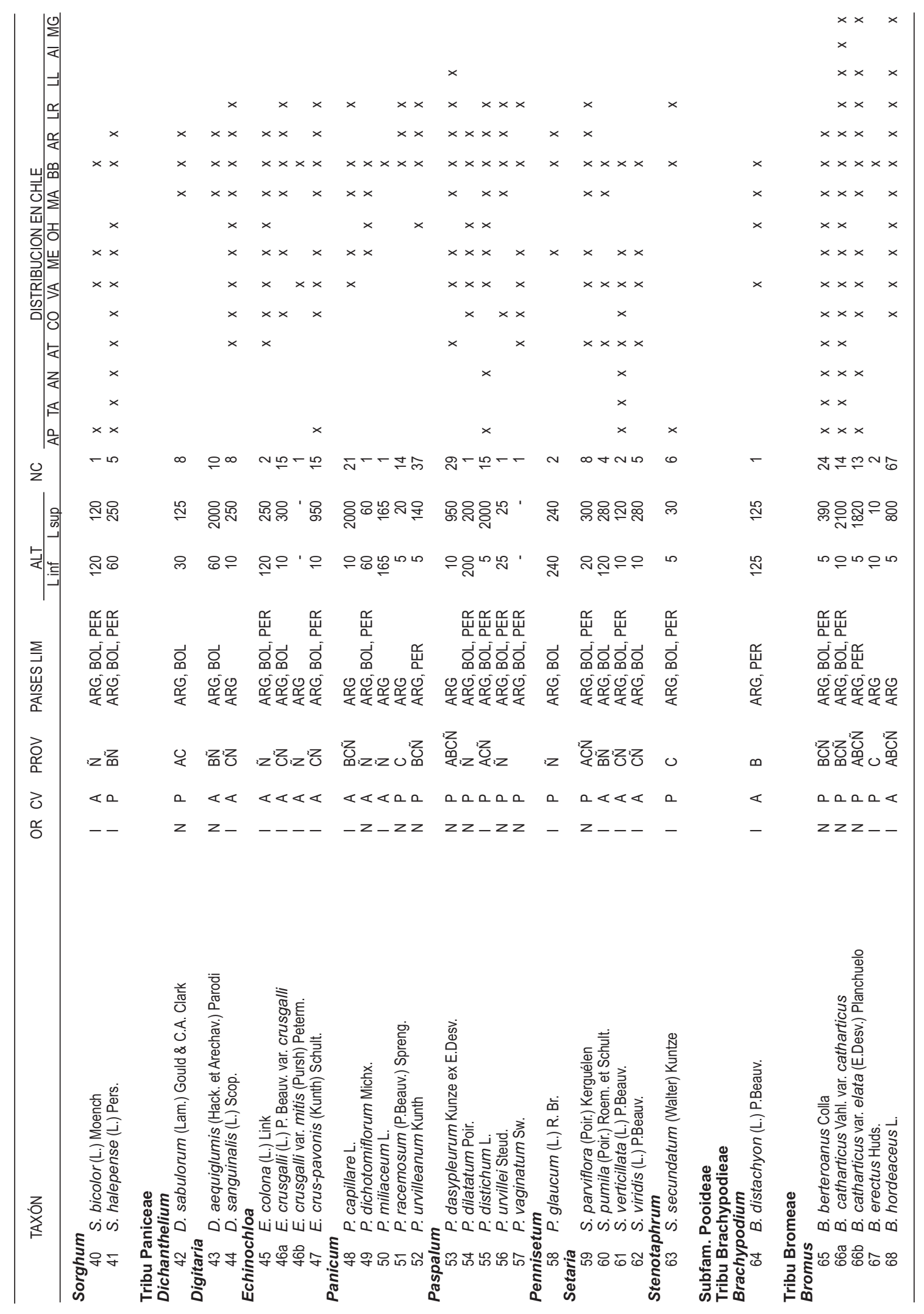


Poaceae de la Región del Bío-Bío, Chile: Finot, V.L. ET AL.

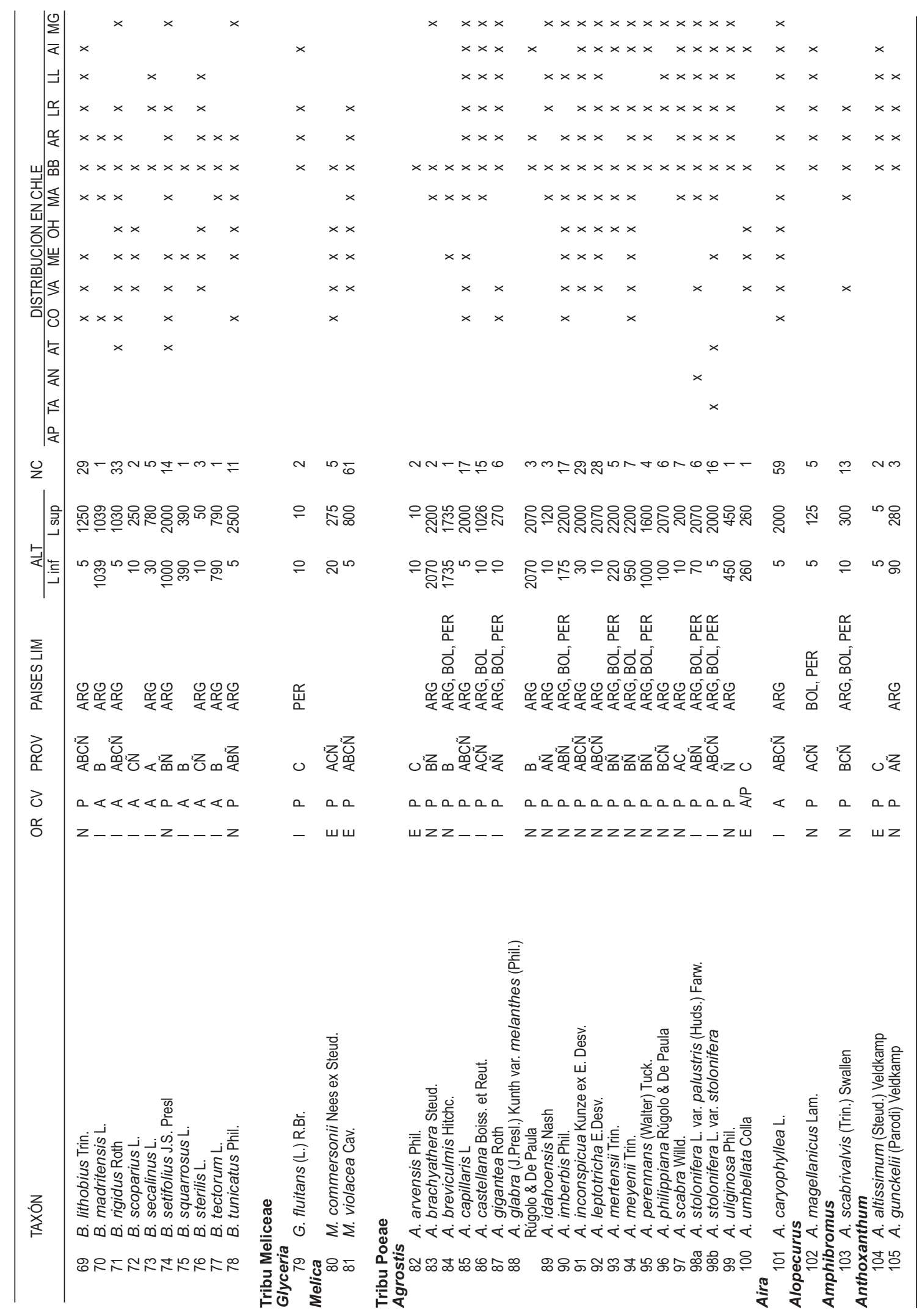


Gayana Bot. 66(2), 2009

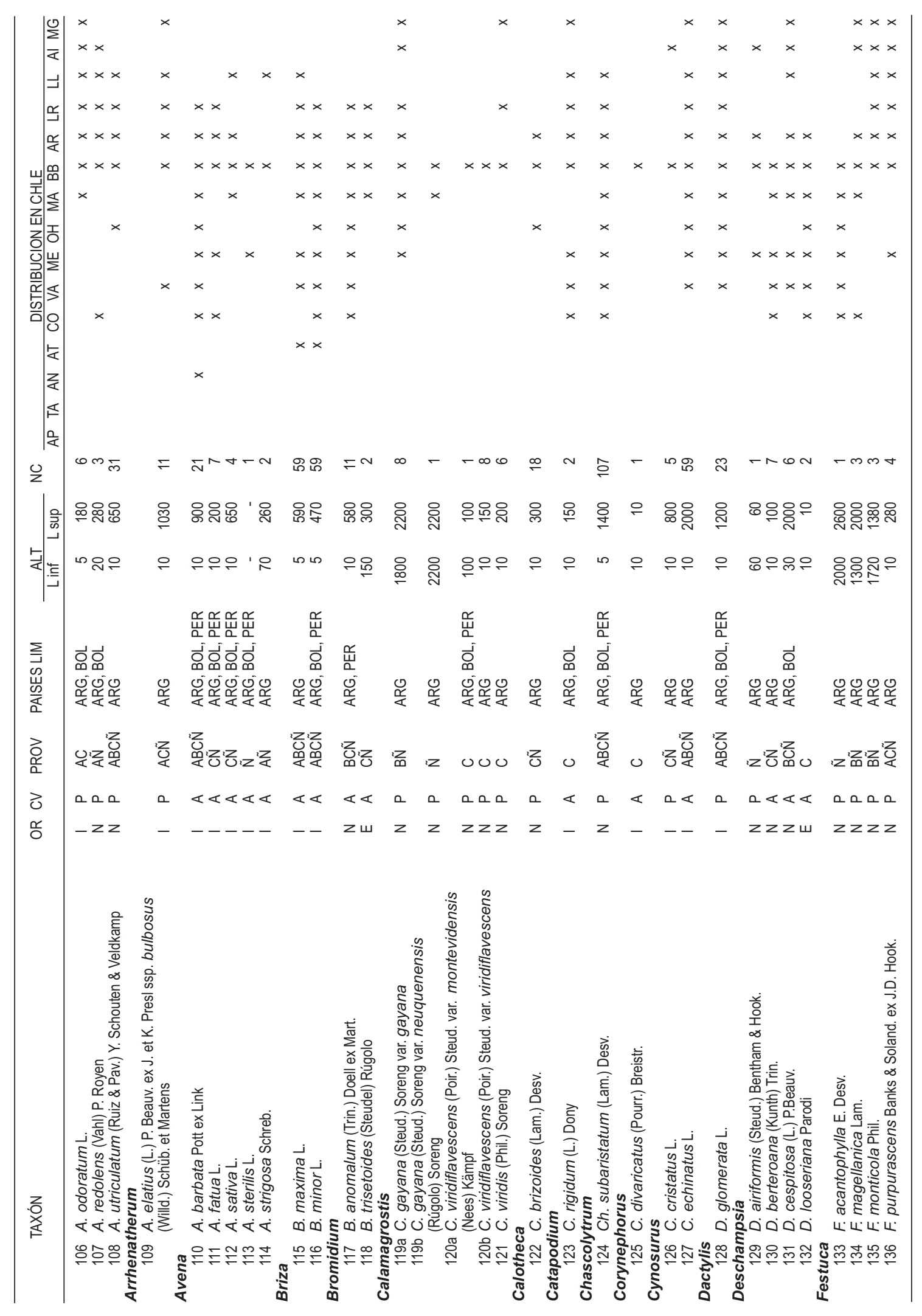


Poaceae de la Región del Bío-Bío, Chile: Finot, V.L. ET AL.

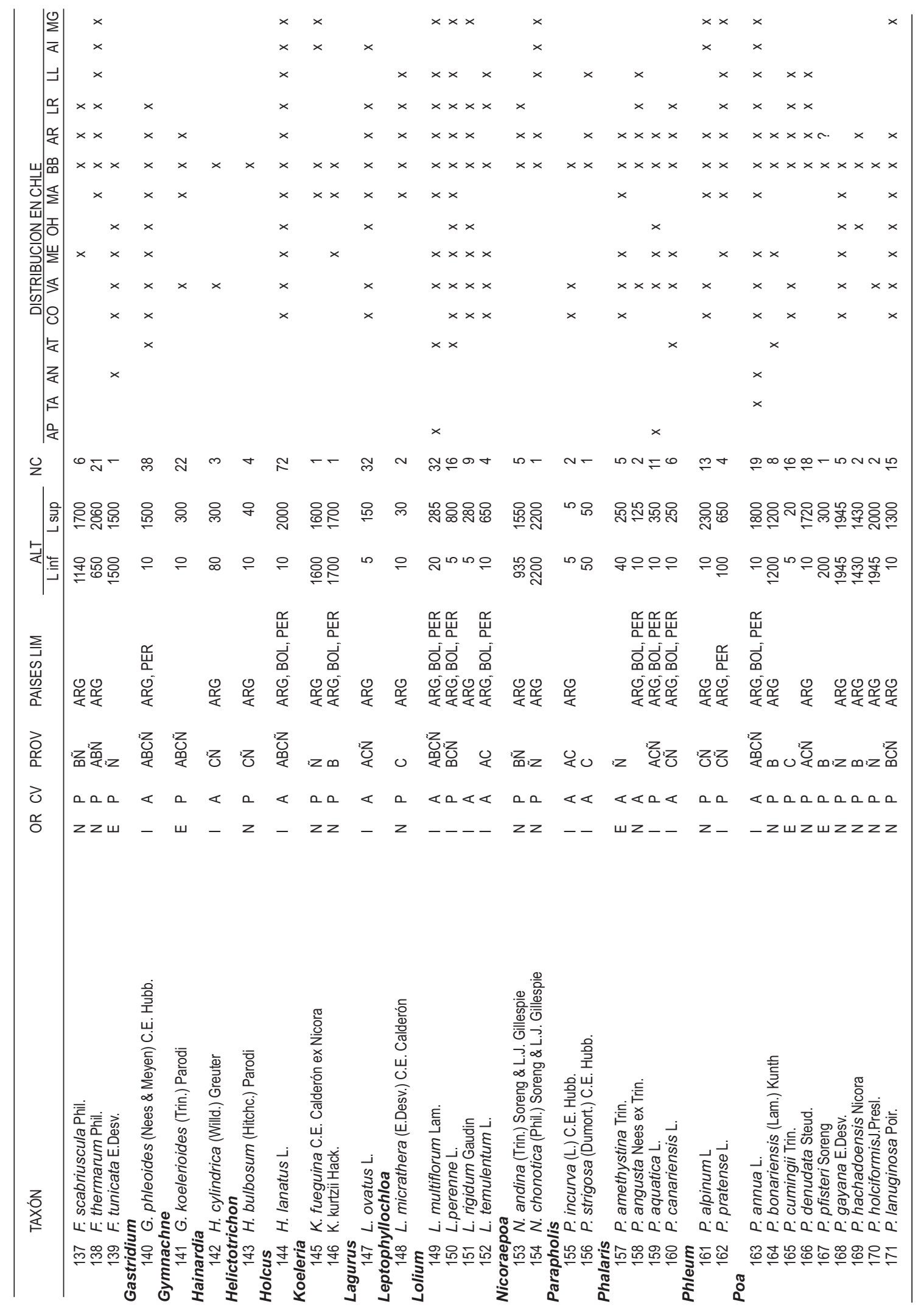


Gayana Bot. 66(2), 2009

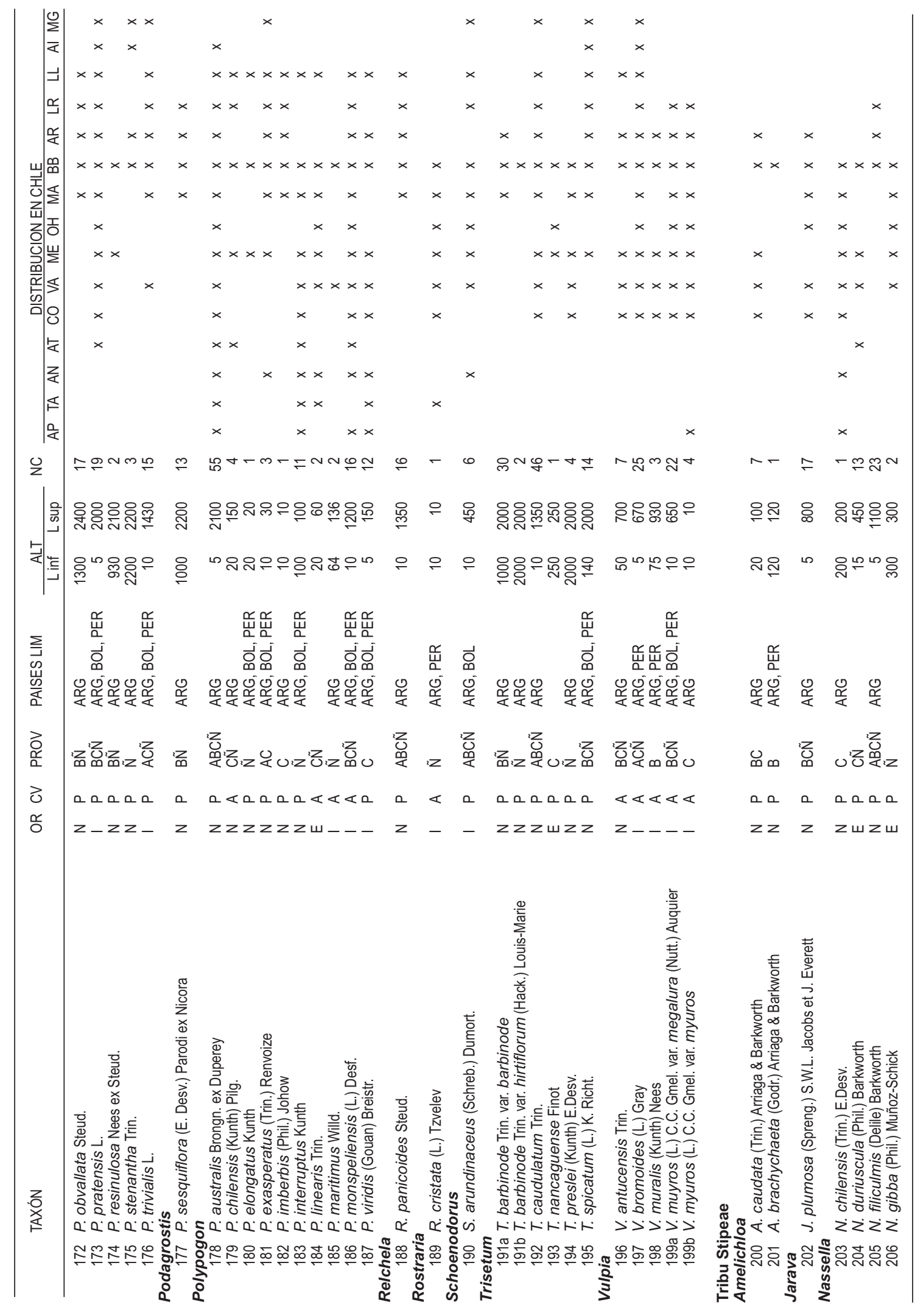


Poaceae de la Región del Bío-Bío, Chile: Finot, V.L. ET AL.

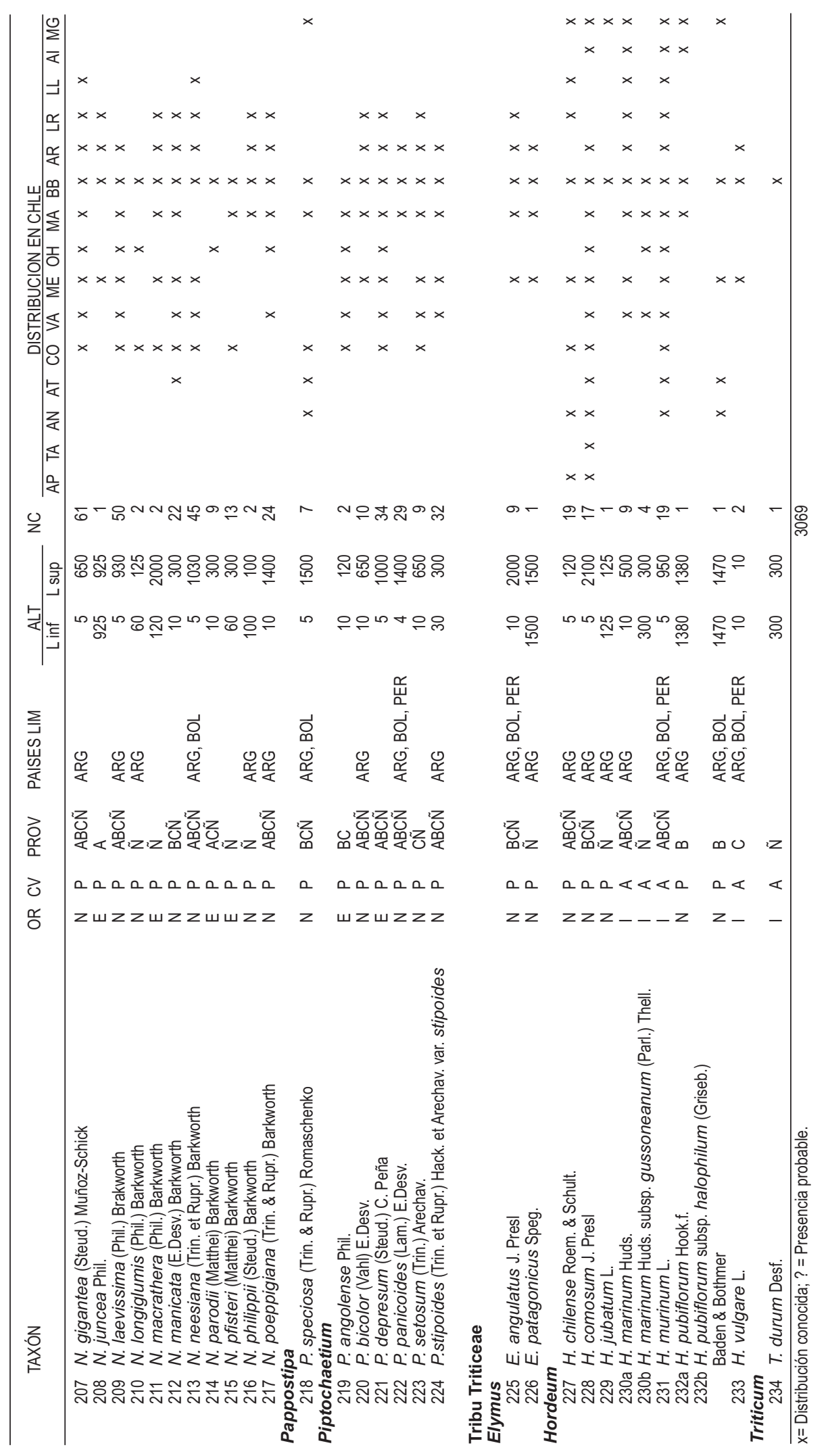


Gayana Bot. 66(2), 2009

TABLA II. Número de tribus, géneros y especies de las subfamilias de Poaceae registrados en la Región del Bío-Bío, Chile.

TABLE II. Number of tribes, genera and species of the subfamilies of Poaceae registered in the region of Bío-Bío.

\begin{tabular}{lccccc}
\hline Subfamilia & Tribus & \multicolumn{2}{c}{ Géneros } & \multicolumn{2}{c}{ Especies } \\
\hline Aristidoideae & 1 & $\mathrm{n}^{\mathbf{0}}$ & $\%$ & $\mathrm{n}^{\mathbf{0}}$ & $\%$ \\
Arundinoideae & 1 & 1 & 1,32 & 2 & 0,85 \\
Bambusoideae & 1 & 1 & 1,32 & 1 & 0,43 \\
Chloridoideae & 1 & 6 & 7,89 & 11 & 2,14 \\
Danthonioideae & 1 & 3 & 3,95 & 14 & 4.70 \\
Ehrhartoideae & 1 & 1 & 1,32 & 1 & 0,43 \\
Panicoideae & 2 & 12 & 15,79 & 29 & 12,39 \\
Pooideae & 6 & 51 & 67,11 & 171 & 73,08 \\
Total & 14 & 76 & 100 & 234 & 100 \\
\hline
\end{tabular}

La Fig. 4 muestra la distribución de la riqueza de especies en la Región. La mayor diversidad de especies se encontró en las cuadrículas 15 , 24, 25 (Concepción y alrededores, con 50, 127 y 48 especies, respectivamente), situadas en la zona del bosque caducifolio de Concepción, actualmente casi completamente reemplazado por plantaciones de Pinus radiata D.Don (Gajardo 1994); las cuadrículas 17, 18 (Chillán, con 43 y 72 especies respectivamente), situadas en la Depresión Intermedia, con espinal de Acacia caven como vegetación dominante (Stolpe 2006),

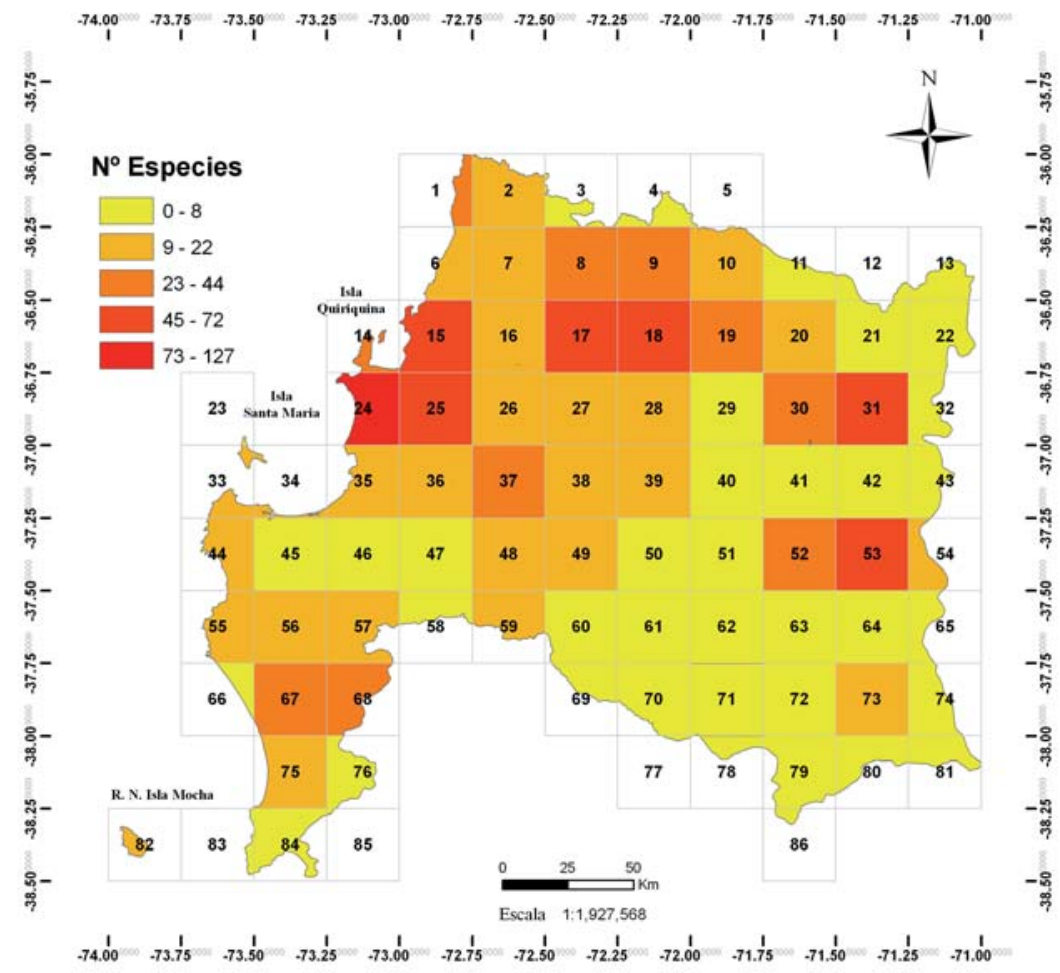

Figura 4. Número de especies por cuadrícula $0,25^{\circ}$ x $0,25^{\circ}$ en la Región del Bío-Bío.

Figure 4 . Number of species per $0.25^{\circ}$ x $0.25^{\circ}$ grid cell the Region of Bío-Bío. 
también altamente intervenida por la agricultura y la forestación, especialmente con Eucalyptus globulus Labill.; las cuadrículas 30, 31 (cordillera de Chillán, con 43 y 63 especies respectivamente) con bosque caducifolio alto-andino de Chillán y bosque de roble y raulí (bosque caducifolio andino de Bío-Bío) y la cuadrícula 53 (P.N. Laguna del Laja, con 49 especies), donde junto con el bosque caducifolio andino de Bío-Bío domina la estepa alto-andina boscosa (Gajardo 1994). Dieciséis cuadrículas no han sido colectadas y, en consecuencia, no registran especies presentes.

\section{ORIGEN FITOGEOGRÁFICO}

De las 234 especies determinadas, 155 (66,2\%) son nativas, de las cuales un $11.5 \%$ (27 especies) son endémicas de Chile, un 54,7\% (128 especies) son nativas no endémicas y un $33,7 \%$ (79 especies) son introducidas (Tabla III). Sólo cuatro subfamilias reúnen las especies introducidas en la región. De las 79 especies introducidas determinadas, la subfamilia Pooideae comprende la mayor parte (58 especies, 73,41\%), seguida por Panicoideae (14 especies, 17,72\%), Chloridoideae (6 especies, $7,60 \%$ ) y Ehrhartoideae (1 especie, 1,27\%). De las especies nativas no endémicas, cerca de un $70 \%$ (91 especies) pertenece a Pooideae, un
$11,75 \%$ (15 especies) a Panicoideae y un 9,38\% (12 especies) a Danthonioideae. Ehrhartoideae no presenta especies nativas ni endémicas en Chile. Por su parte, Aristidoideae y Arundinoideae sólo comprenden especies nativas. En Chloridoideae el número de especies se reparte equitativamente entre nativas (5 especies) e introducidas (6 especies). A nivel de áreas protegidas, el mayor número de especies introducidas se encontró en el SN Península de Hualpén (26 especies, 45\%). Porcentajes relativamente altos se encontraron también en Isla Mocha, donde de un total de 16 especies registradas, nueve $(56 \%)$ son plantas introducidas (Tabla IV). Especies nativas presentes también en otros países, pero que de Chile sólo se tienen registros para esta región del país, son Calamagrostis viridiflavescens (Poir.) Steud. var. viridiflavescens, C. viridiflavescens var. montevidensis (Nees) Kämpf, Helictotrichon bulbosum (Hitchc.) Parodi y Trisetum barbinode Trin. var. hirtiflorum (Hack.) Louis-Marie, presentes también en Argentina (Parodi 1949). Helictotrichon bulbosum sólo habita en Chile y Argentina (Parodi 1949) y es el único representante del género Helictotrichon en el hemisferio sur; en Chile es una especie rara, considerada por algunos autores como una especie de Trisetum (Finot 2003).

TABLA III. Número de especies nativas, endémicas e introducidas de las subfamilias de Poaceae registradas en la Región del Bío-Bío, Chile.

TABLE III. Number of native, endemic and introduced species of the subfamilies of Poaceae registered in the Region of Bío-Bío.

\begin{tabular}{lcccccccc}
\hline & \multicolumn{2}{c}{ Nativas } & \multicolumn{2}{c}{ Endémicas } & \multicolumn{2}{c}{ Introducidas } & \multicolumn{2}{c}{ Total } \\
\cline { 2 - 9 } Subfamilia & $\mathrm{N}^{\mathrm{o}}$ & $\%$ & $\mathrm{~N}^{\mathrm{o}}$ & $\%$ & $\mathrm{~N}^{\mathrm{o}}$ & $\%$ & $\mathrm{~N}^{\mathrm{o}}$ & $\%$ \\
\hline Aristidoideae & 2 & 0,86 & 0 & 0,00 & 0 & 0,00 & 2 & 0,86 \\
Arundinoideae & 1 & 0,43 & 0 & 0,00 & 0 & 0,00 & 1 & 0,43 \\
Bambusoideae & 3 & 1,28 & 2 & 0,86 & 0 & 0,00 & 5 & 2,14 \\
Chloridoideae & 5 & 2,14 & 0 & 0,00 & 6 & 2,56 & 11 & 4,70 \\
Danthonioideae & 11 & 4,70 & 3 & 1,28 & 0 & 0,00 & 14 & 5,98 \\
Ehrhartoideae & 0 & 0,00 & 0 & 0,00 & 1 & 0,43 & 1 & 0,43 \\
Panicoideae & 15 & 6,41 & 0 & 0,00 & 14 & 5,98 & 29 & 12,39 \\
Pooideae & 91 & 38,9 & 22 & 9,40 & 58 & 24,8 & 171 & 73,08 \\
\hline Total & 128 & 54,7 & 27 & 11,5 & 79 & 33,7 & 234 & 100 \\
\hline
\end{tabular}


Gayana Bot. 66(2), 2009

TABLA IV. Número de especies presentes en zonas protegidas por el SNASPE.

TABLE IV. Number of species in SNASPE's protected areas.

\begin{tabular}{lcccc}
\hline Área & Nativas & Endémicas & Introducidas & Total \\
\hline PN Laguna Laja & 47 & 2 & 15 & 64 \\
RN Isla Mocha & 7 & 0 & 9 & 16 \\
RN Ñuble & 4 & 0 & 2 & 6 \\
RN Ralco & 16 & 1 & 3 & 20 \\
SN Península de Hualpén & 24 & 8 & 26 & 58 \\
SN Lobería de Cobquecura & 8 & 2 & 12 & 22 \\
\hline
\end{tabular}

De las especies introducidas, Cynosurus cristatus L. y Corynephorus divaricatus (Pourr.) Breistr. han sido colectadas hasta ahora sólo en la región del Bío-Bío. Corynephorus divaricatus, introducida en Chile, ha sido señalada sólo para esta Región del país, no habiéndosele registrado nuevamente desde su introducción en 1838. Por otra parte, especies cuya presencia no había sido documentada en esta región del país son Bromus madritensis, $B$. tectorum, Festuca acanthophylla, F. monticola, F. purpurascens, F. tunicata y Rostraria cristata.

\section{ENDEMISMOS}

Las especies endémicas de Chile que habitan la Región del Bío-Bío se distribuyen en 3 subfamilias: Bambusoideae, Danthonioideae y Pooideae. De las cinco especies de Bambusoideae determinadas, dos son endémicas de Chile (Chusquea cumingii y Ch. quila). Por su parte, Danthonioideae incluye tres especies endémicas de Chile en la Región del Bío-Bío: Danthonia araucana, D. chilensis var. aureofulva y Rytidosperma quirihuense. De la subfamilia Pooideae, 22 especies son endémicas de Chile (Tabla III). El género con mayor número de especies endémicas es Nassella (6 especies): $N$. duriuscula, N. gibba, N. juncea Phil., N. macrathera, $N$. parodii y $N$. pfisteri. Sólo 45 cuadrículas presentaron especies endémicas (Fig. 5, Tabla V). La mayor parte de las especies endémicas se encuentran en las cuadrículas 24 (14 especies) y 15 (7 especies) (Concepción y alrededores); estas dos cuadrículas reúnen 14 de las 27 especies endémicas detectadas en la Región: Agrostis arvensis, Anthoxanthum altissimum, Bromidium trisetoides, Chusquea quila, Danthonia araucana,
D. chilensis var. aureofulva, Deschampsia looseriana, Gymnachne koelerioides, Melica commersonii, M. violacea, Nassella duriuscula, Piptochaetium angolense, P. depresum y Polypogon linearis. Las especies endémicas más ampliamente distribuidas son Chusquea quila y Melica violacea (19 cuadrículas). A nivel de áreas protegidas, el mayor número de especies endémicas se encontró en el Santuario Hualpén ( 8 especies), en tanto en las restantes áreas el número de endémicas fluctúa entre 0 y 2 especies (Tabla IV). Endémicas a nivel regional son Agrostis arvensis, que se conoce solamente por el material original colectado por C. Gay en 1839, en Concepción, y Poa pfisteri, recientemente descrita (Soreng \& Peterson 2008) sobre material colectado por A. Pfister en 1943, en Santa Bárbara.

\section{Ciclo DE VIDA}

La gran mayoría de las especies registradas (168 especies, 71,8\%) son plantas perennes, de las cuales 140 son nativas y 28 introducidas. Por el contrario, la gran mayoría de las especies introducidas (54 especies, 23\%), son anuales, en tanto sólo 12 especies anuales son nativas. De entre las especies endémicas, sólo Agrostis umbellata, Bromidium trisetoides, Deschampsia looseriana, Phalaris amethystina y Polypogon linearis son anuales.

\section{DisTRIBUCIÓN ALTITUDINAL}

La distribución altitudinal de las especies (Fig. 6) muestra que la mayor parte de ellas se distribuye por debajo de los $250 \mathrm{~m}$ s. n. m. El número de especies disminuye consistentemente con la altitud. Tanto las especies nativas como las introducidas muestran 
Poaceae de la Región del Bío-Bío, Chile: Finot, V.L. ET AL.

esta tendencia, aunque estas últimas tienden a desaparecer por sobre los $2.000 \mathrm{~m}$. Las especies de Chusquea (Bambusoideae) tienden a concentrarse por sobre los $1.600 \mathrm{~m}$, con la excepción de $C h$. valdiviensis que alcanza sólo los $800 \mathrm{~m}$, aunque la mayor parte de las especies fue también colectada a bajas altitudes (Tabla I). Tienden a crecer por sobre los 650 y hasta los $2.100 \mathrm{~m}$ las especies de
Cortaderia (Danthonioideae). Sólo C. selloana ha sido colectada a bajas altitudes. En general, las especies de Pooideae tienden a concentrarse en las altitudes más bajas, aunque algunas especies como Festuca acantophylla, F. thermarum, Nicoraepoa chonotica, Poa holciformis, P. stenantha, Trisetum barbinode y $T$. preslei tienden a crecer sólo por sobre los $1600 \mathrm{~m}$.

Tabla V. Distribución de las especies de Poaceae endémicas de Chile, en las cuadrículas de 0,25 x $0,25^{\circ}$.

TABLE V. Distribution of Chilean endemic Poaceae in the $0.25 \times 0.25^{\circ}$ grid cells.

\begin{tabular}{|c|c|c|}
\hline $\mathrm{N}^{\mathrm{o}}$ & Especie & Cuadrículas \\
\hline 1 & Agrostis arvensis & 24 \\
\hline 2 & Agrostis umbellata & 16 \\
\hline 3 & Anthoxanthum altissimum & 24,25 \\
\hline 4 & Bromidium trisetoides & 8,24 \\
\hline 5 & Chusquea cumingii & 30 \\
\hline 6 & Chusquea quila & $21,24,30,31,33,34,35,44,45,47,55,56,57,62,66,67,68,71,75$ \\
\hline 7 & Danthonia araucana & $14,17,18,24,27,35,55,57,68,76$ \\
\hline 8 & Danthonia chilensis var. aureofulva & $2,3,7,8,9,15,16,17,49$ \\
\hline 9 & Deschampsia looseriana & 15,24 \\
\hline 10 & Festuca tunicata & 30 \\
\hline 11 & Gymnachne koelerioides & $3,8,15,24,25,38,55$ \\
\hline 12 & Melica commersonii & $2,7,15,24,68$ \\
\hline 13 & Melica violacea & $1,6,8,10,15,17,18,24,25,26,27,37,38,39,42,48,49,59,60$ \\
\hline 14 & Nassella duriuscula & $2,15,16,20,24,25,37$ \\
\hline 15 & Nassella gibba & 9 \\
\hline 16 & Nassella juncea & 57 \\
\hline 17 & Nassella macrathera & 18,31 \\
\hline 18 & Nassella parodii & $8,9,17,24,26$ \\
\hline 19 & Nassella pfisteri & $8,9,17,18$ \\
\hline 20 & Phalaris amethystina & 3,25 \\
\hline 21 & Piptochaetium angolense & 15,59 \\
\hline 22 & Piptochaetium depressum & $1,24,49$ \\
\hline 23 & Poa cumingii & 24 \\
\hline 24 & Poapfisteri & 53 \\
\hline 25 & Polypogon linearis & 8,24 \\
\hline 26 & Rytidosperma quirihuense & 1 \\
\hline 27 & Trisetum nancaguense & 26 \\
\hline
\end{tabular}



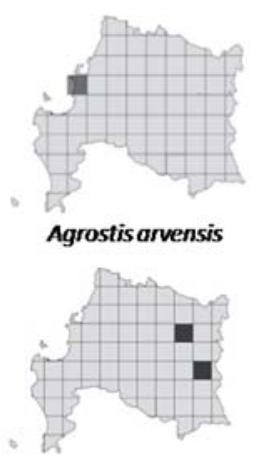

Chusquea cumingï

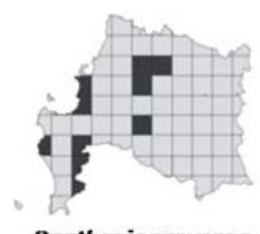

Danthonia araucana

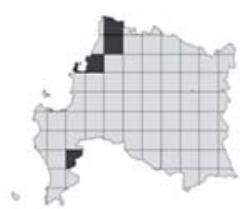

Melica commersonï

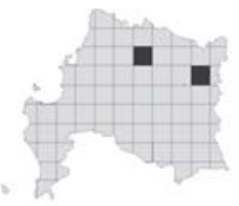

Nassella macrathera

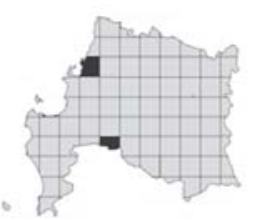

Piptochaetiumangolense

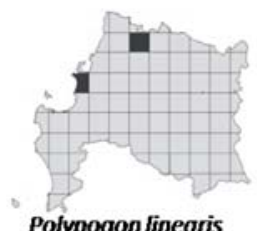

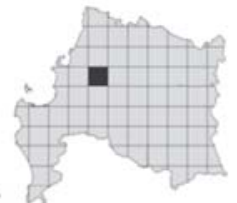

Agrostis umbellata
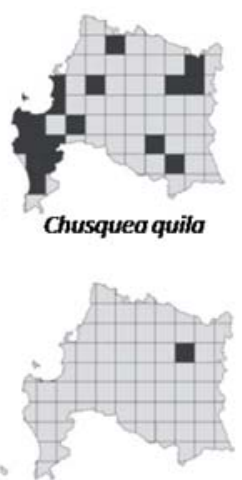

Festucatunicata

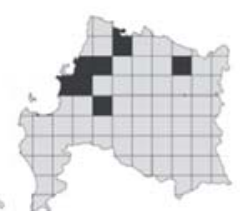

Nassella duriuscula

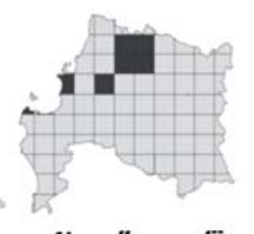

Nassella parodii

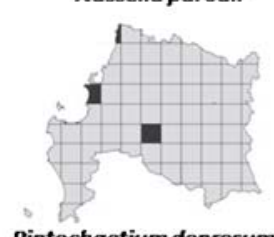

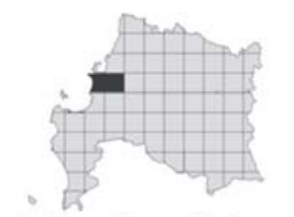

Anthoxanthum aftissimum

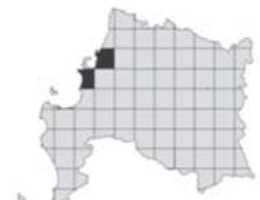

Deschampsia looseriana

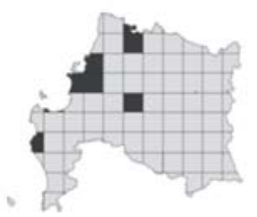

Gymnachne koelerioides

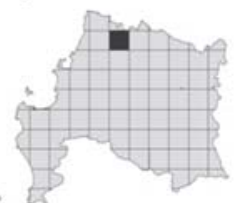

Nassellagibba

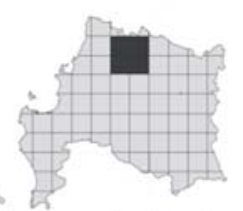

Nassellapfisterii

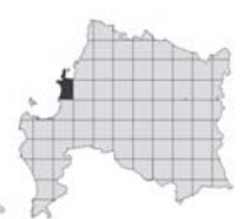

Poacumingii
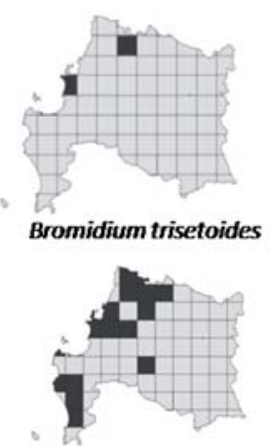

Danthonia chilensis var. aureofulva

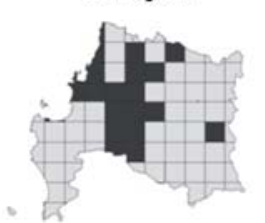

Melica violacea

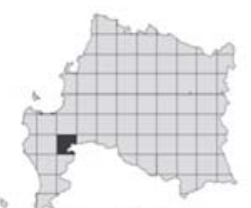

Nassella juncea
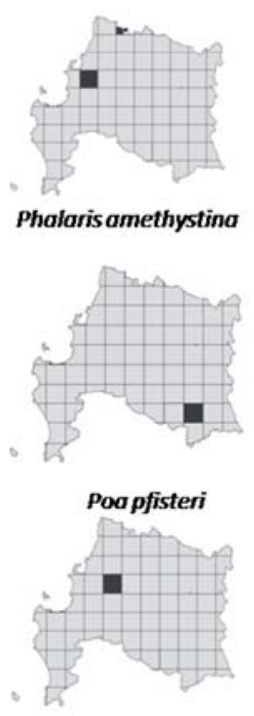

Trisetum nancaguense

Figura 5. Distribución de las taxa de Poaceae endémicas de Chile, en la Región del Bío-Bío.

Figure 5. Distribution of the Chilean endemic taxa Poaceae, in the Region of Bío-Bío. 


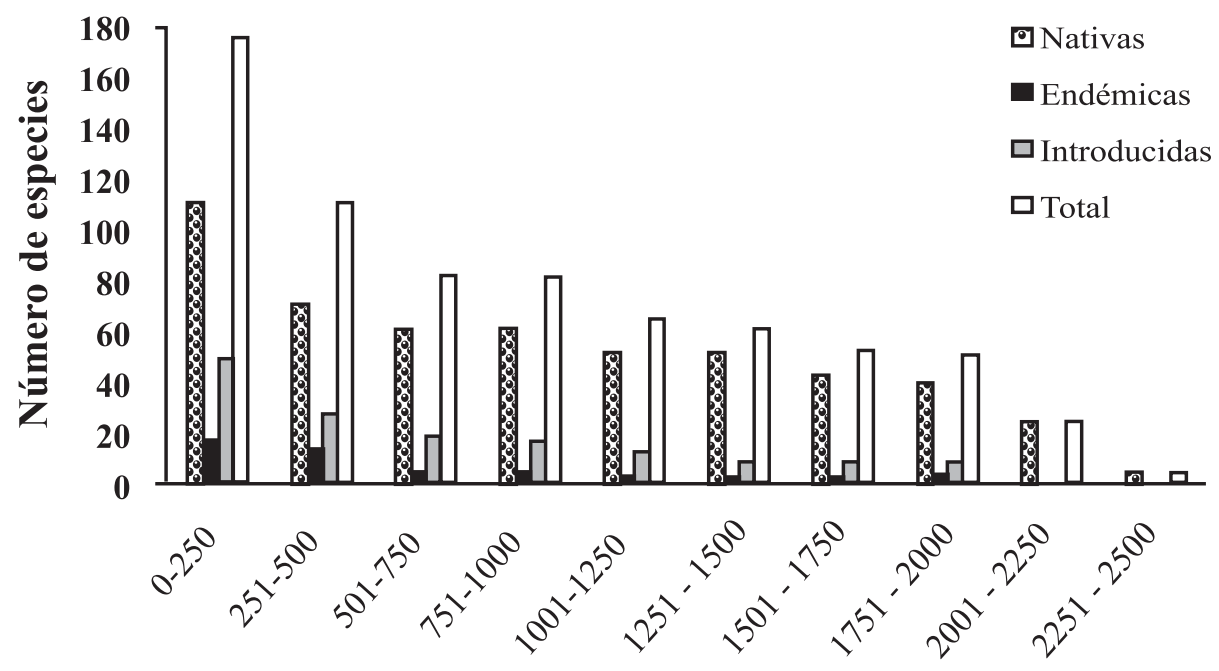

Altitud (m s.n.m)

FiguRa 6. Distribución altitudinal de gramíneas nativas, endémicas e introducidas, en la Región del Bío-Bío.

Figure 6. Altitudinal distribution of native, endemic and introduced grasses in the Region of Bío-Bío.

\section{DISCUSION}

Los análisis de la biodiversidad, de la distribución de la riqueza específica a distintos niveles (nacional, regional), el conocimiento de las zonas que albergan especies endémicas, la detección de zonas cuya conservación es necesaria y otros muchos temas relacionados con el conocimiento y conservación de la biodiversidad, requieren de un importante esfuerzo de recolección para que las bases de datos organizadas lleguen a ser lo más completas que sea posible. Ello es necesario pues, como se demuestra en este trabajo, evidencias de la existencia de más del $60 \%$ de las especies de Poaceae que habitan en la Región del Bío-Bío fueron obtenidas sólo a partir de 1918 (durante el segundo de los dos siglos considerados), cuando el esfuerzo de recolección aumentó considerablemente.

Las curvas de acumulación de especies son elementos importantes, pues consideran la rareza o abundancia de una especie en la zona investigada (Koellner et al. 2004). La función de Clench aplicada a las curvas de rarefacción es uno de los modelos paramétricos más utilizados para estimar, a partir de estas curvas, la riqueza específica (Arroyo et al. 1998, León et al. 1998).
Los modelos no paramétricos, como los índices de Chao (Chao 1984), son tal vez preferibles, pues no asumen un tipo particular de distribución de los datos (Escalante 2003). En el presente trabajo, con los datos de recolección de especies en el tiempo, ambos métodos llegan a resultados similares, indicando que entre un 85 y $88 \%$ de la riqueza específica estimada está representada en el inventario disponible. La lectura inversa es que aún es posible, aumentando el esfuerzo de recolección, incrementar en cerca de 30 a 40 el número de especies de Poaceae a nivel regional. Es, en consecuencia, de enorme importancia que se otorgue a los herbarios nacionales los medios necesarios para incrementar el esfuerzo de recolección. El conocimiento de la distribución de la intensidad de recolección permite identificar áreas de interés botánico así como las áreas no recolectadas.

La división de la región en cuadrículas es un buen método para detectar cuáles son las áreas no recolectadas o escasamente recolectadas y planificar futuros viajes de recolección (Marticorena et al. 1998a). Es posible que el número de especies por cuadrícula se relacione más con el número de recolectas por cuadrícula que con factores bióticos 
o abióticos, aunque el número total de especies disminuye con la altitud, de forma monotónica, en la región estudiada. Aunque en algunas cuadrículas no se registraron especies recolectadas, algunas de ellas incluyen una escasa superficie (cuadrículas 5, 77, 78, 81, 83 y 85). De acuerdo con Linder (2001), la elección del tamaño de la grilla puede afectar fuertemente los resultados. Una consecuencia de la disminución del tamaño de las cuadrículas es el aumento del número de cuadrículas artificialmente vacías, es decir, donde la especie está presente pero no fue registrada. Aunque el aumento en el tamaño de las cuadrículas podría disminuir el número de cuadrículas "vacías", se corre el riesgo de perder resolución en el análisis (Linder 2001).

Las 234 especies de 76 géneros identificadas representan, aproximadamente, un $61 \%$ de los géneros y un $43 \%$ de las especies de Poaceae presentes en Chile. De éstas, 27 son endémicas de Chile $(11,5 \%)$, las que se concentran principalmente en dos áreas: 1. La zona costera (Concepción y alrededores, cuadrículas 15 y 24), que reúne la mayor riqueza específica de la región así como la mayor cantidad de especies de Poaceae endémicas de Chile, comprendida en la zona del bosque caducifolio de Concepción, actualmente muy alterado. 2. La zona de Quirihue y alrededores (cuadrícula 8), cubierta por espinal de Acacia caven, que aunque con menor riqueza de especies de Poaceae, reúne siete especies endémicas de Chile; esta zona ha sido considerada un sitio de importancia para la conservación de la biodiversidad (Muñoz-Schick et al. 1996).

Una parte importante del contingente florístico analizado lo constituyen, sin embargo, las especies introducidas (79 especies, 33,7\%). Para Chile, Zuloaga et al. (2008) cita 148 especies de gramíneas introducidas, esto es, aproximadamente un $27 \%$ del total de especies de Poaceae que habitan el territorio continental. En consecuencia, alrededor de un 53\% de las especies de gramíneas introducidas, voluntaria o involuntariamente en Chile, está presente en la Región del Bío-Bío, lo que se relaciona, probablemente, con el alto grado de intervención antrópica que presenta esta región del país y donde se comportan como malezas agrestales o ruderales (Matthei 1995, Finot 1997). Algunas de ellas, sin embargo, se han hecho escasas (e.g. Lolium temulentum) debido a las intensas medidas de control. Otra especie introducida escasa es Corynephorus divaricatus, cuya presencia fue dada a conocer para la Provincia de Concepción
(Rúgolo de Agrasar 1999) y de la que sólo se conoce un ejemplar recolectado por Claudio Gay en 1838 (SGO). Es posible que tras su introducción (probablemente involuntaria), la especie no pudiera naturalizarse en el país; no obstante, se incluyó en el listado.

La metodología empleada en este trabajo se puede aplicar, en trabajos futuros, también a otras regiones del país. Es necesario profundizar también los estudios sobre la distribución y diversidad de las especies introducidas o invasivas y su relación con la actividad antrópica (agricultura, plantaciones forestales, industrialización, etc.), así como aumentar las recolecciones de Poaceae (y probablemente también de otros grupos de plantas) en áreas protegidas. En el caso de la Región del Bío-Bío, existen zonas como la R.N. Ñuble con muy pocas especies recolectadas (6 especies) o como los Huemules de Niblinto, donde aún no hay recolectas $\mathrm{y}$, asimismo, existen cuadrículas en zonas no protegidas que no arrojaron especies, posiblemente debido a su difícil accesibilidad.

\section{AGRADECIMIENTOS}

Los autores agradecen al Sr. Manuel Oyarzún, Dpto. de Suelos y Recursos Naturales, Universidad de Concepción, su colaboración en la elaboración de los mapas. A Alicia Marticorena y a los revisores anónimos por sus valiosas sugerencias para mejorar este trabajo. A la Dirección de Investigación de la Universidad de Concepción, el financiamiento otorgado a través del Proyecto DIUC 204.121.009-1.0.

\section{BIBLIOGRAFIA}

AceVedo de Vargas, R. 1959. Las especies de Gramineae del género Cortaderia en Chile. Boletín del Museo Nacional de Historia Natural 27: 205-246.

Arriaga, M.O. \& M.E. Barkworth. 2006. Amelichloa: A new genus in the Stipeae (Poaceae). Sida 22: $145-149$.

Arroyo, M.T.K. 1999. Criterios e indicadores para la conservación de la biota en ecosistemas mediterráneos. Revista Chilena de Historia Natural 72: 473-474.

Arroyo, M.T.K., C. Marticorena, P. Miranda, O. Matthei, A. Landero \& F. SQueo. 1989. Contribution to the high elevation flora of the Chilean Patagonia: A checklist of species on mountains on an East-West transect in the 
Sierra de los Baguales, Latitude $50^{\circ} \mathrm{S}$. Gayana, Botánica 46: 121-151.

Arroyo, M.T.K., C. Marticorena \& M. Muñoz-Schick. 1990. A checklist of the native annual flora of continental Chile. Gayana, Botánica 47: 119-135.

Arroyo, M.T.K., Ch. von Bohlen, L. Cavieres \& C. Marticorena. 1992. Survey of the alpine flora of Torres del Paine National Park, Chile. Gayana, Botánica 49: 47-70.

Arroyo, M.T.K., C. Castor, C. Marticorena, M. Muñoz, L. Cavieres, O. Matthei, F.A. Squeo, M. Grosjean \& R. RodríGuez. 1998. La flora del parque nacional Llullaillaco ubicado en la zona de transición de las lluvias de invierno-verano en los Andes del norte de Chile. Gayana Botánica 55(2): 93-110.

BAeza, C.M. 1996. Los géneros Danthonia DC. y Rytidosperma Steud. (Poaceae) en América-Una revisión. Sendtnera 3: 11-93.

Baeza, C.M., C. Marticorena \& R. Rodríguez. 1999. Catálogo de la flora vascular del Monumento Natural Contulmo, Chile. Gayana Botánica 56: 125-135.

Baeza, C.M., C. Marticorena, T. Stuessy, E. Ruiz \& M. Negritto. 2007. Poaceae en el Archipiélago de Juan Fernández (Robinson Crusoe). Gayana Botánica 64(2): 125-174.

Baeza, C.M., T.F. Stuessy \& C. Marticorena. 2002. Notes on the Poaceae of the Robinson Crusoe (Juan Fernández) Islands, Chile. Brittonia 54 (3): $154-163$.

Bette, A. L., P.M. Jørgensen, T. Consiglio, I. JimÉnez, J.G. Blake, L.G. Lohmann \& O.M. Montiel. 2009. Predicting species distributions from herbarium collections: does climate bias in collection sampling influence model outcomes? Journal of Biogeography 35: 105-116.

Снао, A. 1984. Non-parametric estimation of the number of class in a population. Scandinavian Journal of Statistics 11: 265-270.

Cialdella, A.M. \& M.O. Arriaga. 1998. Revisión de las especies sudamericanas del género Piptochaetium (Poaceae, Poöideae, Stipeae). Darwiniana 36(14): $105-157$

Cialdella, A.M. \& L.M. Giussani. 2002. Phylogenetic relations of the genus Piptochaetium (Poaceae: Poöideae, Stipeae): Evidence from morphological data. Annals of the Missouri Botanical Garden 89: 305-336

Colwell, R.K. 2006. EstimateS 8.0. Statistical estimation of species richness and shared species from samples. Software and User's Guide. http://purl. oclc.org/estimates.

Colwell, R.K., C.X. Mao \& J. Chang. 2004. Interpolating, extrapolating, and comparing incidence-based species accumulation curves. Ecology 85: 2717-2727.

De Paula, M.E. 1975. Las especies de Hierochlöe (Gramineae) de Argentina y Chile. Darwiniana 19: 422-457.

Di Castri, F. \& E. Hajek. 1976. Bioclimatología de
Chile. Ediciones Pontificia Universidad Católica de Chile, Santiago, Chile.128 pp.

Di Rienzo J.A., F. Casanoves, M.G. Balzarini, L. Gonzalez, M. Tablada \& C.W. Robledo. 2009. InfoStat versión 2009. Grupo InfoStat, FCA, Universidad Nacional de Córdoba, Argentina

Domínguez, E., E. ElvebakK, C. Marticorena \& A. Pauchard. 2006. Plantas introducidas en el Parque Nacional Torres del Paine, Chile. Gayana Botánica 63(2): 131-141.

Escalante, T. 2003. ¿Cuántas especies hay? Los estimadores no paramétricos de Chao. Elementos 52: 53-56.

Escalante, T., J. Llorente, D.N. Espinosa \& J. Soberón. 2000. Bases de datos y sistemas de información: aplicaciones en biogeografía. Revista de la Academia Colombiana de Ciencias 24: 325-341.

EsRI. 1996. Software ARCVIEW-GIS 3.2. Environmental Systems Research Institute, NY. 340 pp.

FinOt, V.L. 1997. Estudio florístico de las malezas de la provincia de Nuble. Agro-Ciencia 13: 203-216.

FinOt, V. L. 2003. Trisetum. In: R. Soreng, J., P. M. Peterson, G. Davidse, E. J. Judziewicz, F. O. Zuloaga, T. S. Filgueiras, and O. Morrone. 2003. Catalogue of New World grasses (Poaceae): IV. subfamily Pooideae. Pp. 659-676. Contributions from the United States National Herbarium 48: $1-730$.

Finot, V.L., P.M. Peterson, F.O. Zuloaga, R.J. Soreng \& O. Matthei. 2005. A revision of Trisetum (Poaceae: Pooideae: Aveninae) in South America. Annals of the Missouri Botanical Garden 93: 533-568.

FunK, V.A., M.F. Zermoglio \& N. Nasir. 1999. Testing the use of specimen collection data and GIS in biodiversity exploration and conservation decision making in Guyana. Biodiversity and Conservation 8: 727-751.

Gajardo, R. 1994. La vegetación natural de Chile. Clasificación y distribución geográfica. Editorial Universitaria, Santiago de Chile, 165 pp.

Grass Phylogeny Working Group (GPWG). 2001. Phylogeny and subfamilial classification of the grasses (Poaceae). Annals of the Missouri Botanical Garden 88: 373-457.

Нiтcнсоск, A.S. 1927. The grasses of Ecuador, Peru and Bolivia. Contributions from the United States National Herbarium 24: 291-556.

IREN. 1979. Fragilidad de los ecosistemas naturales de Chile. Volumen 1. http://biblioteca digital.ciren. $\mathrm{cl} /$ gsdlexterna/collect/bdirenci/index/assoc/ HASH0138.dir/II04079V1a.pdf [consulta 3 junio 2009].

JimÉnez-Valverde, A. \& J. Hortal. 2003. Las curvas de acumulación de especies y la necesidad de evaluar la calidad de los inventarios biológicos. Revista Ibérica de Aracnología 8: 151-161.

Judziewicz, E.J., R.J. Soreng, G. Davidse, P.M. Peterson, T.S. Filgueiras \& F.O. Zuloaga. 2000. Catalogue of New World Grasses (Poaceae): I. Subfamilies Anomochloideae, Bambusoideae, Ehrhartoideae, 
and Pharoideae. Contributions from the United States National Herbarium 39: 1-128.

Klopper, R.R., L. Gautier, C. Chatelain, G.F. Smith \& R. SPICHIGER. 2007. Floristics of the angiosperm flora of Sub-Saharan Africa: an analysis of the African plant checklist and database. Taxon 56(1): 201-208.

Koellner, T., A.M. Hersperger \& T. Wohlmemuth. 2004. Rarefaction method for assessing plant species diversity on a regional scale. Ecography 27: 532-544.

KunKel, G. 1968. Die Phanerogamen der Insel La Mocha (Chile). Willdenowia 4(3): 329-400.

León, J.L., J. Soberón \& J. Llorente. 1998. Assessing completeness of Mexican sphinx moth inventories through species accumulation functions. Diversity and Distributions 4: 37-44.

LINDER, H.P. 2001. Plant diversity and endemism in subSaharan tropical Africa. Journal of Biogeography 28: $169-182$

Marticorena, C. 1990. Contribución a la estadística de la flora vascular de Chile. Gayana, Botánica 47: $85-113$.

Marticorena, C., O. Matthei, R. Rodríguez, M.T.K. Arroyo, M. Muñoz, F. Squeo \& G. Arancio. 1998a. Catálogo de la flora vascular de la Segunda Región (Región de Antofagasta), Chile. Gayana, Botánica 55: 23-83.

Marticorena, C. \& M. Quezada. 1985. Catálogo de la flora vascular de Chile. Gayana, Botánica 42(12): 1-147.

Marticorena, C., T.F. Stuessy \& C.M. Baeza. 1998 b. Catalogue of the vascular flora of the Robinson Crusoe or Juan Fernández, Chile. Gayana, Botánica 55: 187-211.

MatTHeI, O. 1965. Estudio crítico de las gramíneas del género Stipa en Chile. Gayana, Botánica 13: 1-137.

Matthei, O. 1975. Der Briza-Komplex in Südamerika: Briza, Calotheca, Chascolytrum, Poidium (Gramineae). Willdenowia 8: 1-168.

Matthei, O. 1986. El género Bromus L. (Poaceae) en Chile. Gayana, Botánica 43: 47-100.

Matthei, O. 1987a. Las especies del género Aristida L. (Poaceae) en Chile. Gayana, Botánica 44: 17-23.

Matthei, O. 1987b. Las especies del género Panicum (Poaceae) en Chile. Gayana, Botánica 44: 25-32.

Matthei, O. 1995. Manual de las malezas que crecen en Chile. Alfabeta Impresores, Santiago de Chile, $545 \mathrm{pp}$.

Matthei, O., C. Marticorena \& T.F. Stuessy. 1993. La flora adventicia del Archipiélago de Juan Fernández. Gayana Botánica 50: 69-102.

Moreira-MuÑoz, A. \& M. MuÑOZ-SCHICK. 2007. Classification, diversity, and distribution of Chilean Asteraceae: implications for biogeography and conservation. Biodiversity Research 13: 818-828.

Moreno, C.E. \& G. Halffter. 2000. Assessing the completeness of bat inventories using species accumulation curves. Journal of Applied Ecology 37: 149-158.

Morrone, O., S.S. Aliscioni \& F.O. Zuloaga. 2005.
Análisis de la diversidad y distribución geográfica de la familia Poaceae en la provincia de Jujuy, Argentina. Annals of the Missouri Botanical Garden 92: 595-639.

MuÑoz, C. 1941. Índice bibliográfico de las gramíneas chilenas. Boletín Técnico $\mathrm{N}^{\mathrm{o}}$ 2, Ministerio de Agricultura, Santiago de Chile, $88 \mathrm{pp}$.

MuÑoz-Schick, M. 1985. Revisión de las especies del género Melica L. (Gramineae) en Chile. Boletín del Museo Nacional de Historia Natural 40: 41$81+9$ figs.

MuÑoz-Schick, M. 1990. Revisión del género Nassella (Trin.) E.Desv. (Gramineae) en Chile. Gayana, Botánica 47: 9-35.

MuÑoz-SCHICK, M., H. NúÑEz \& J. YÁÑEz. 1996. Libro rojo de los sitios prioritarios para la conservación de la diversidad biológica en Chile. Ministerio de Agricultura, Corporación Nacional Forestal, Santiago de Chile, Chile.

Myers, N., R.A. Mittermeier, C.G. Mittermeier, G.A.B. DE Fonseca \& J. Kent. 2000. Biodiversity hotspots for conservation priorities. Nature 403: 853-858.

Nicora, E.G. 1978. Gramineae. En: M.N. Correa (ed.), Flora Patagónica 3: 1-563. Colecciones Científicas Inta, Buenos Aires.

NicoRA, E.G. 1998. Revisión del género Eragrostis Wolf. (Gramineae: Eragrostidae) para Argentina y países limítrofes. Boissera 54: 1-109.

Nicora, E. G. \& Z. Rúgolo de Agrasar. 1987. Los géneros de Gramíneas de América Austral, Argentina, Chile, Uruguay, y áreas limítrofes de Bolivia, Paraguay y Brasil. 1-611. Ediciones Hemisferio Sur S.A., Buenos Aires.

PArodi, L.R. 1949. Los géneros de Avéneas de la flora argentina. Revista Argentina de Agronomía 16: 205-223.

Peñailillo, P. 1996. Anatherostipa: un nuevo género de Poaceae (Stipeae). Gayana Botánica 53: 277-284.

Peterson, P.M., R.J. Soreng, G. Davidse, T.S. Filgueiras, F.O. Zuloaga \& E. Judziewicz. 2001. Catalogue of New World Grasses (Poaceae): II. Subfamily Chloridoideae. Contributions from the United States National Herbarium 41: 1-255.

Pysek, P. 2003. How reliable are data on alien species in Flora Europaea? Flora 198: 499-507.

Pysek, P., D.M. Richardson, M. RejMÁnek, G.L. Webster, M. Williamson \& J. Kirschner. 2004. Alien plants in checklists and floras: towards better communication between taxonomists and ecologists. Taxon 53(1): 131-143.

Reiche, C. 1903. La isla de la Mocha. Anales del Museo Nacional de Chile. Pp.: 1-104.

Renvoize, S.A. 1998. Gramíneas de Bolivia. The Royal Botanical Garden, Kew, 644 pp.

Rodríguez, R., J. Grau, C. Baeza \& A. Davies. 2008a. Lista comentada de las plantas vasculares de los Nevados de Chillán, Chile. Gayana Botánica 65(2): 153-197.

Rodríguez, R., A. Marticorena \& E. Teneb. 2008 b. Plantas vasculares de los ríos Baker y Pascua, 
región de Aisén, Chile. Gayana Botánica 65(1): 39-70.

Romaschenko, K., P.M. Peterson, R.J. Soreng, N. García-Jacas, O. Futorna \& A. Susanna. 2008. Molecular phylogenetics analysis of the American Stipeae (Poaceae) resolves Jarava sensu lato polyphyletic: evidence for a new genus, Pappostipa. Journal of the Botanical Research Institute of Texas 2: 165-192.

Rúgolo de Agrasar, Z.E. 1978. Las especies australes del género Deyeuxia Clar. (Gramineae) de la Argentina y Chile. Darwiniana 21: 417-453.

Rúgolo DE Agrasar, Z.E. 1982. Revalidación del género Bromidium Nees et Meyen emend. Pilger (Gramineae). Darwiniana 24: 187-216.

Rúgolo DE Agrasar, Z.E. 1999. Corynephorus (Gramineae), género adventicio para la flora de Chile. Hickenia 2: 299-302.

Rúgolo deAgrasar, Z.E. \&A.M. Molina. 1997. Las especies del género Agrostis L. (Gramineae: Agrostideae) de Chile. Gayana Botánica 54(2): 91-156.

Rundel, P.W., M.O. Dillon \& B. Palma. 1996. Flora and vegetation of Pan de Azúcar National Park in the Atacama desert of northern Chile. Gayana, Botánica 53(2): 295-315.

SÁnchez-Ken, J.G., L.G. Clark, E.A. Kellogg \& E.E. KAY. 2007. Reinstatement and emendation of subfamily Micrairoideae (Poaceae). Systematic Botany 32: 71-80.

Scholz, H. 2006. Kikuyuochloa, genus novum (Poaceae: Paniceae). Feddes Repertorium 117: 512-518.

Schmidt, M., H. Kreft, A. Thiombiano \& G. ZizKa. 2005. Herbarium collections and field data-based plant diversity maps for Burkina Faso. Diversity and Distributions 11: 509-516.

Smith, G.F. \& M.M. Wolfson. 2004. Mainstreaming biodiversity: the role of taxonomy in bioregional planning activities in South Africa. Taxon 53(2): 467-468.

Soberón, J. \& J. Llorente. 1993. The use of species accumulation functions for the prediction of species richness. Conservation Biology 7: 480-488.

Soreng, R.J. \& L.J. Gillespie. 2007. Nicoraepoa (Poaceae: Poeae), a new South American genus based on Poa subg. Andinae, and emmendation of Poa sect. Parodiochloa of the Sub-Antarctic islands. Annals of the Missouri Botanical Garden 94: 821-849

Soreng, R.J. \& P.M. Peterson. 2008. New records of Poa (Poaceae) and Poa pfisteri: a new species endemic to Chile. Journal of Botanical Research Institute of Texas 2: 847-859.

Soreng, R.J., P.M. Peterson, G. DAvidse, E. Judziewicz, F.O. Zuloaga, T.S. Filgueiras \& O. Morrone. 2003. Catalogue of New World
Grasses (Poaceae): IV. Subfamily Pooideae. Contributions from the United States National Herbarium 48: 1-730.

Stolpe, N. 2006. Descripciones de los principales suelos de la VIII Región de Chile. Publicaciones Dpto. de Suelos y Recursos Naturales, Facultad de Agronomía, Universidad de Concepción, Chillán, 83 pp.

Teillier, S., A.J. Hoffmann, F. SaAvedra \& L. Pauchard. 1994. Flora del Parque Nacional El Morado (Región Metropolitana, Chile). Gayana Botánica 51 (1): 13-47.

Teneb, E., L.A. Cavieres, M.J. Parra \& A. Marticorena. 2004. Patrones geográficos de distribución de árboles y arbustos en la zona de transición climática mediterráneo-templada de Chile. Revista Chilena de Historia Natural 77: 51-71.

Tovar, O. 1957. Las gramíneas de Huancavélica. Primera parte. Memorias del Museo de Historia Natural Javier Prado 6: 1-110.

Tovar, O. 1960. Revisión de las especies peruanas del género Calamagrostis. Memorias del Museo de Historia Natural Javier Prado 11: 1-88.

Veit, H. \& K. GarlefF. 1995. Evolución del paisaje cuaternario y los suelos en Chile central-sur. En: J.J. Armesto, C. Villagrán \& M.T.K. Arroyo (eds.), Ecología de los bosques nativos de Chile. Editorial Universitaria, Santiago de Chile.

Vergara, O.E., V. Jerez \& L.E. Parra. 2006. Diversidad y patrones de distribución de coleópteros en la Región del Bío-Bío, Chile: una aproximación preliminar para la conservación de la diversidad. Revista Chilena de Historia Natural 79: 369-388.

Villagrán, C. \& C. Le-Quesne. 1996. El interés biogeográfico-histórico de Chile Central-Sur: ¿Por qué debemos conservar su biota? En M. Muñoz-Schick, H. Núñez \& J. Yáñez (eds.), Libro rojo de los sitios prioritarios para la conservación de la biodiversidad biológica en Chile, Primera edición, Pp. 160-172. Ministerio de Agricultura, Corporación Nacional Forestal, Santiago de Chile, 203 pp.

Zuloaga, F.O., O. Morrone \& M. Belgrano. 2008. Catálogo de las plantas vasculares del Cono Sur. Volumen 1: Pteridophyta, Gymnospermae, Monocotyledoneae. Monographs in Systematic Botany 107, Missouri Botanical Garden Press, St. Louis, Missouri, USA.

Zuloaga, F.O., O. Morrone, G. Davidse, T.S. Filgueiras, P.M. Peterson, R.J. Soreng \& E. Judziewicz. 2003. Catalogue of New World Grasses (Poaceae): III. Subfamilies Panicoideae, Aristidoideae, Arundinoideae, and Danthonioideae. Contributions from the U.S. National Herbarium 46: 1-662. 